\title{
Unusual Photocyclization of Perfluoro cis-1,2-Dimethyl-1,3- Butadienyl Benzenes as a means to Synthesize Partially Fluorinated Naphthalenes.
}

\author{
Michiharu Yamamoto, Dale C. Swenson, and Donald J. Burton* \\ Department of Chemistry, The University of Iowa, Iowa City, IA 52242, USA
}

\begin{abstract}
:
Photoirradiation of the titled compounds perfluoro-cis-1,2-dimethyl-butadienyl benzenes (1), which were prepared in several steps from perfluorovinyl bromide, results in the formation of the corresponding novel naphthalene derivatives and 1,4dihydronaphthalenes. Isolated 1,1,2-trifluoro-3,4-bis(trifluoromethyl)-1,4dihydronaphthalene (3a) could be converted into 1,2-bistrifluoromethyl-3,4difluoronaphthalene ( $\mathbf{2 a}$ ) by base treatment (DABCO); however, 3a did not lead to 2a by photoreaction, suggesting 3a was not a possible photochemical precursor. Competitive photoreaction studies suggest that varying the substituent on benzene ring (e.g. methyl or trifluoromethyl) does not significantly affect the reaction rate. Presently, this reaction mechanism is not yet clearly understood.
\end{abstract}

Keyword: Photocyclization, perfluoro-cis-1,2-dimethyl-butadienylbenzene, 1,2-difluoro-3,4-bis(trifluoromethyl)naphthalene, photoelimination of HF, and 1,1,2-trifluoro-3,4-bis(trifluoromethyl)-1,4-dihydronaphthalene.

*Corresponding author. Tel.: +1 319335 1363. Fax: +1 3193351270.

E-mail address: mburton105@gmail.com (D.J. Burton).

\section{Introduction}

We have reported that trifluoromethylcopper [1], F-vinyl copper [2,3], and Facetylenic copper [4] reagents could be readily obtained via copper(I) halide metathesis reaction of the corresponding cadmium or zinc reagent (Eq. 1). These 
reagents have found extensive utility in preparative organofluorine chemistry [5].

Terminal F-vinyl copper reagents added to perfluoroalkynes can give extended dienyl copper reagents, which could be functionalized via coupling reactions (Eq. 2) $[6,7]$. The reaction of F-dienyl copper reagents with aryl halides generates coupled products (Eq. 3) [6, 7, 8, 9]. We focused on perfluoro cis-1,2-dimethyl butadienyl benzenes (1) which could be readily prepared from perfluoro-1,3-butadienyl copper (6) reagents (Scheme 1).

$$
\begin{aligned}
& \begin{array}{l}
\mathrm{R}_{\mathrm{F}} \mathrm{CF}=\mathrm{CFX}+\mathrm{M} \frac{\mathrm{DMF}}{\mathrm{RT}-60^{\circ} \mathrm{C}} \quad \mathrm{R}_{\mathrm{F}} \mathrm{CF}=\mathrm{CFMX}+\left(\mathrm{R}_{\mathrm{F}} \mathrm{CF}=\mathrm{CF}\right)_{2} \mathrm{M}+\mathrm{MX}_{2} \underset{\mathrm{RT}}{\stackrel{\mathrm{Cu}(\mathrm{l}) \mathrm{X}}{\longrightarrow} \quad \mathrm{R}_{\mathrm{F}} \mathrm{CF}=\mathrm{CFCu}} \\
\mathrm{X}=\mathrm{Br}, \mathrm{I} \quad \mathrm{M}=\mathrm{Cd}, \mathrm{Zn}
\end{array}
\end{aligned}
$$

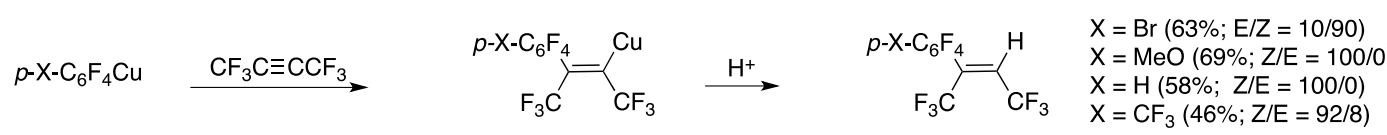

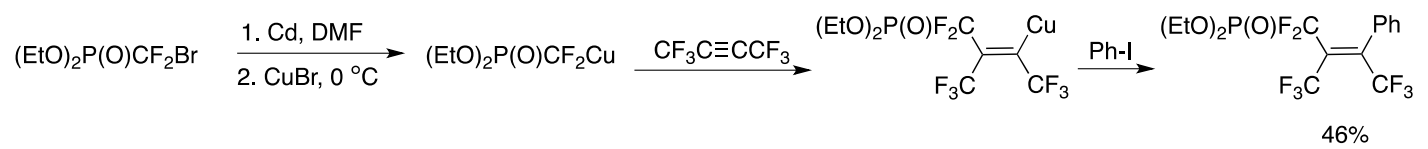

Scheme 1. Synthesis of perfluoro cis-1,2-dimethyl butadienyl benzenes (1)

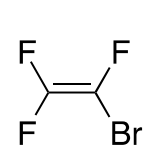

4

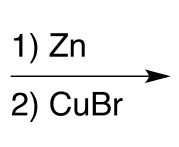<smiles>FC(F)=C(F)F</smiles>

5<smiles>FC(F)=C(F)C(=C(Cl)C(F)(F)F)C(F)(F)F</smiles>

6

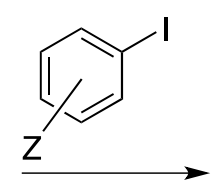

(c)

1

\section{Results and Discussion}

Preparation of $\mathbf{1}$ was carried out by the method [6] shown in Table 1. These transformations were carried out by the one-pot reaction under $\mathrm{N}_{2}$ atmosphere. Conversion of $\mathbf{5}$ to $\mathbf{6}$ was done at room temperature and the yield was calculated by ${ }^{19} \mathrm{~F}-\mathrm{NMR}$ spectroscopy using an internal trifluoromethyl benzene standard. In the conversion of $\mathbf{6}$ to $\mathbf{1}$, a variety of functionalized aryl iodides were added (mole ratio 
0.70 equivalent) to the copper reagent 6 and reaction mixtures were heated at 80 $90^{\circ} \mathrm{C}$ overnight. After reaction, compounds of general structure $\mathbf{1}$ were isolated by silica gel chromatography. The resultant benzenes $\mathbf{1}$ in hexane were photolyzed using a Rayonet reactor at $254 \mathrm{~nm}$ in a quartz tube at $30-40{ }^{\circ} \mathrm{C}$ under degassed conditions (Scheme 2).

Table 1. Preparation of perfluoro-cis-1,2-dimethyl-butadienyl benzenes 1

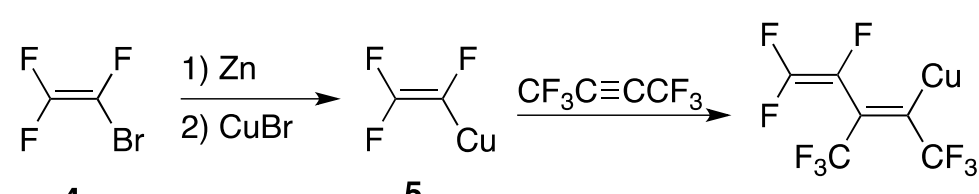

4

5 Aryl Iodide

1b

1c

1d

1e

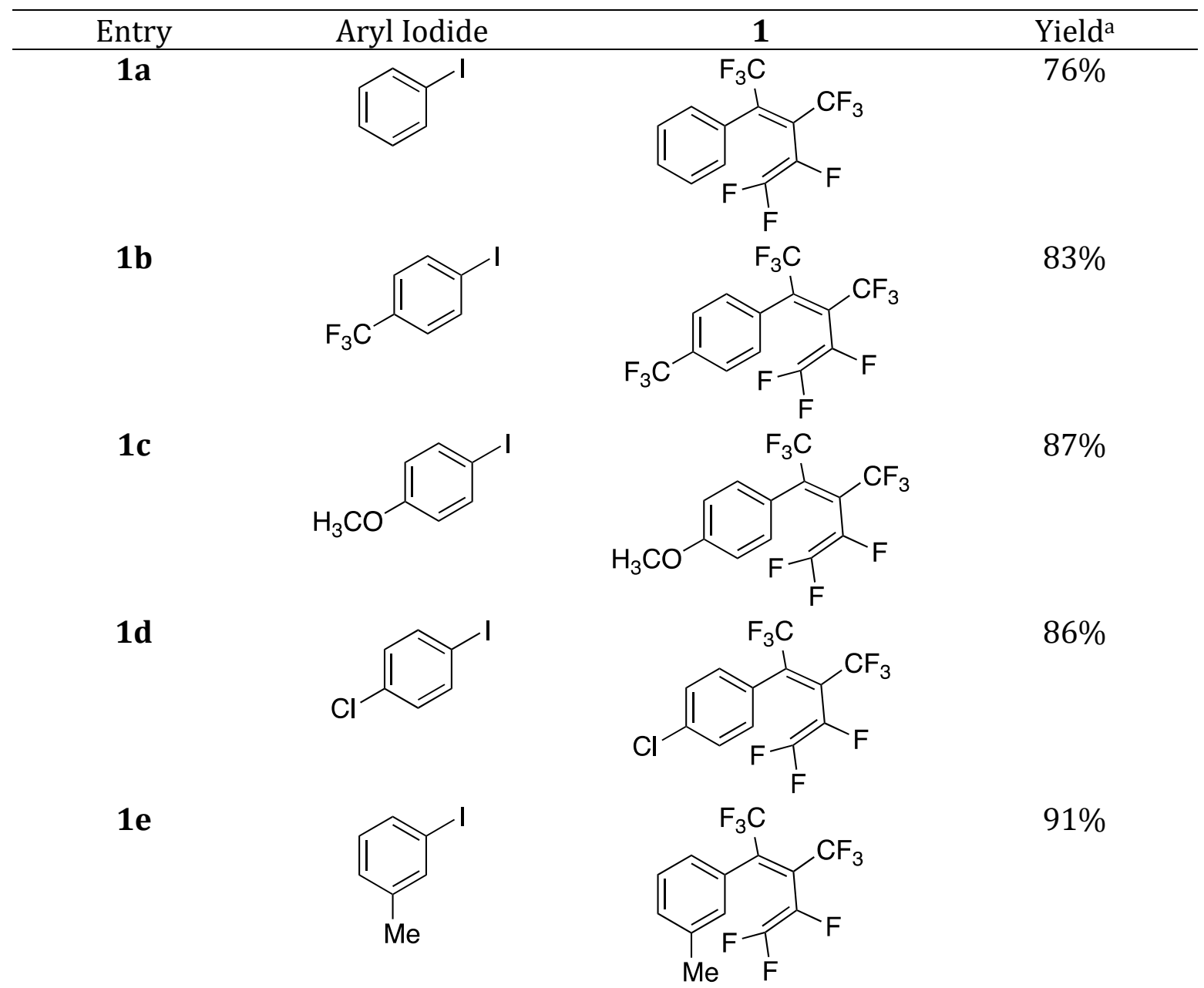

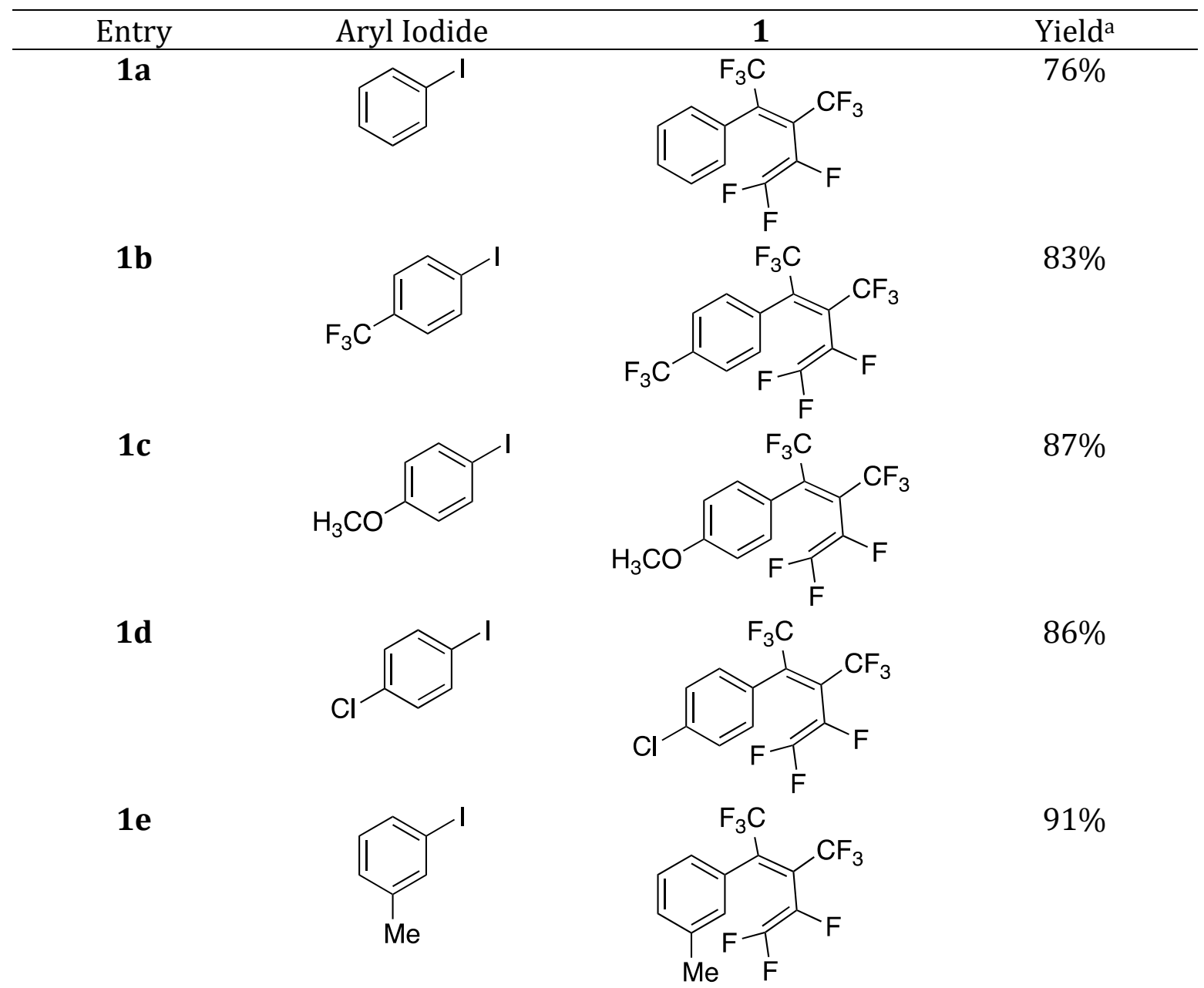

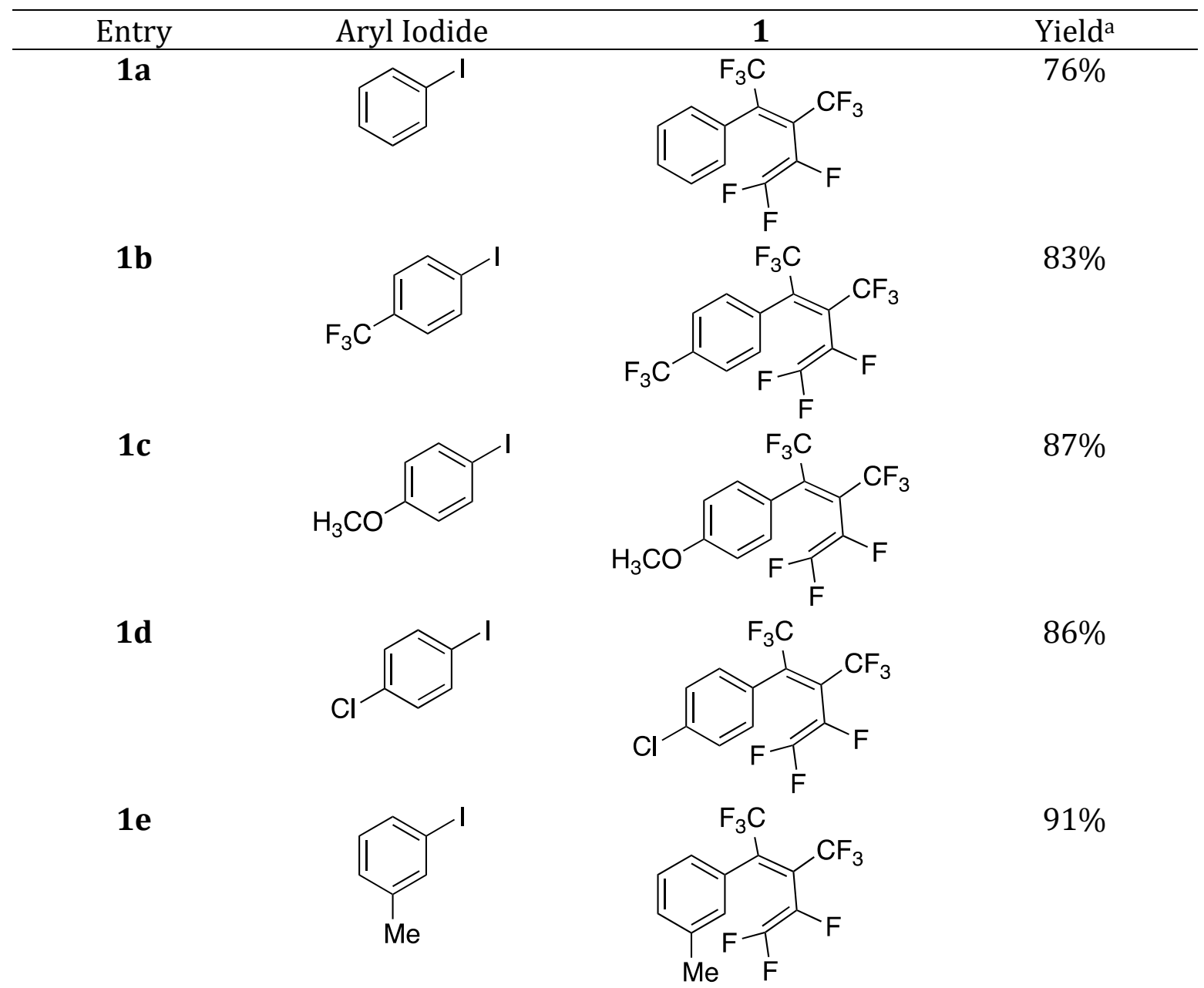

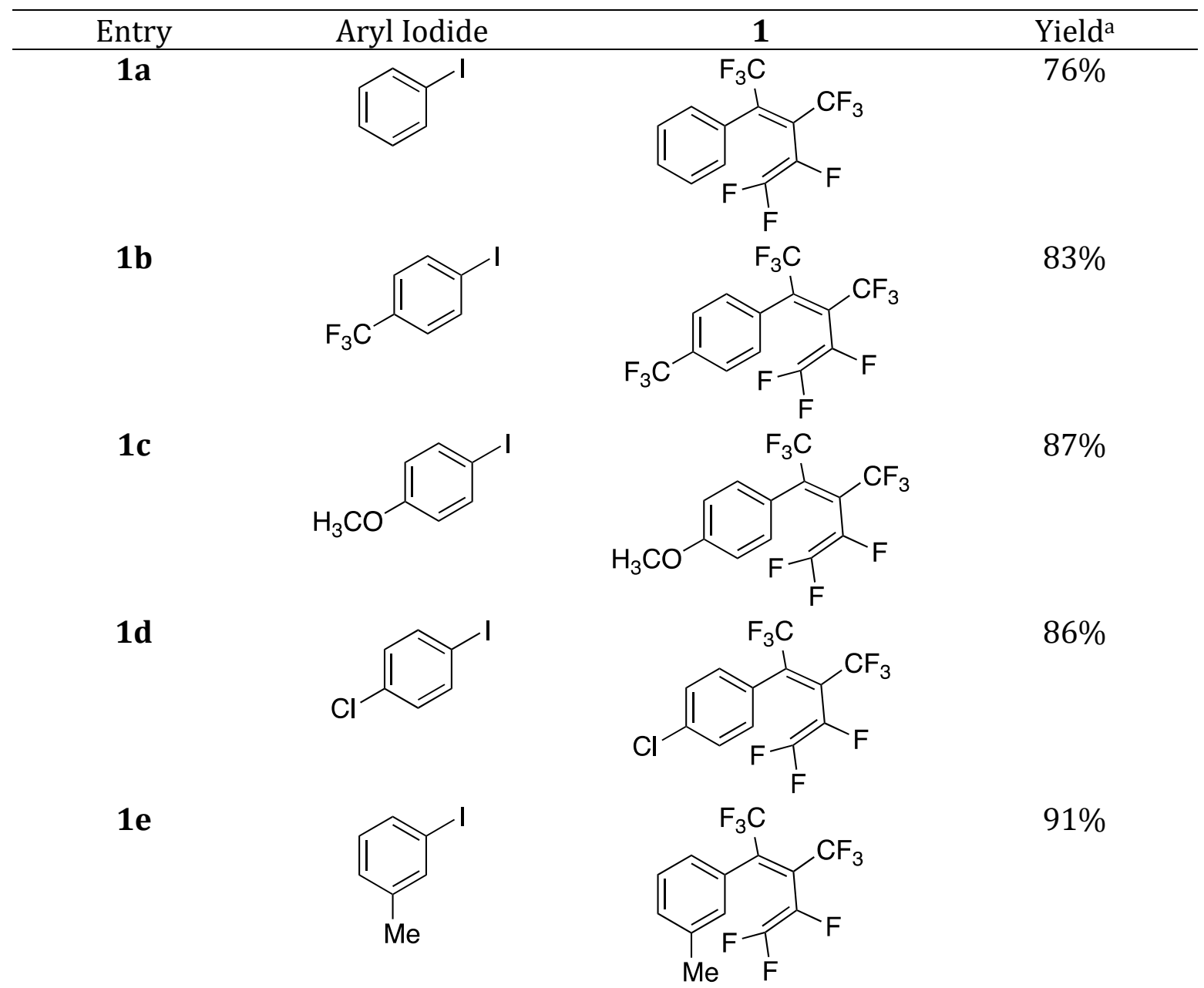

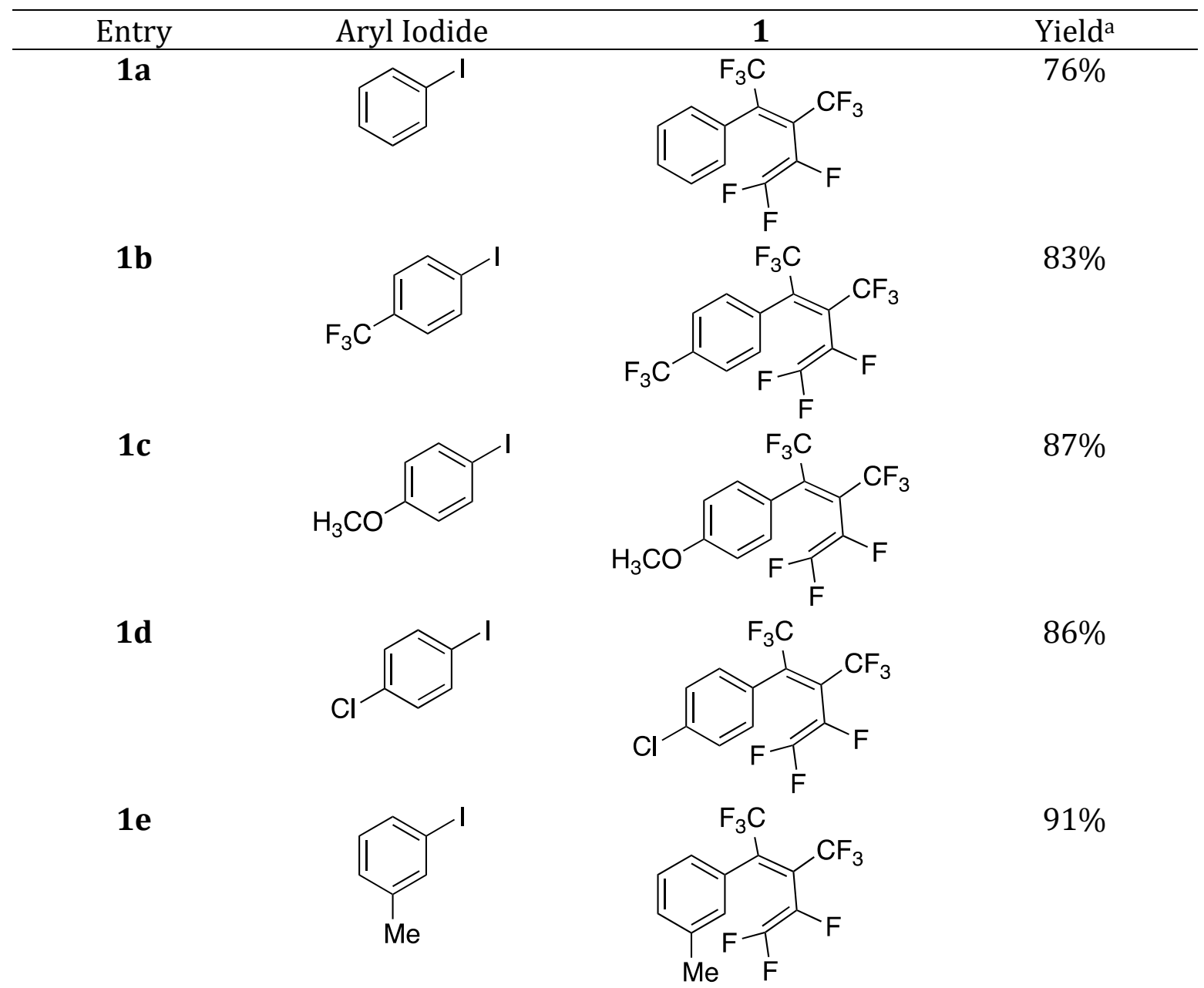

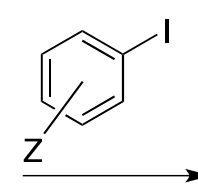

6<smiles>[Z]c1ccc(C(C(=C(C)C)C(F)(F)F)=C(C(F)=C(F)F)C(F)(F)F)cc1</smiles>

1 
<smiles>Ic1ccc(I)cc1</smiles><smiles>FC(F)=C(F)C(=C(C(C(F)=C(F)F)=C(C(F)(F)F)C(F)(F)F)c1ccc(F)cc1)C(=C(C(F)(F)F)C(F)(F)F)C(F)(F)F</smiles>

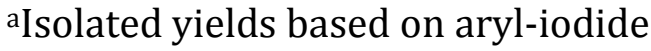

Scheme 2. Synthesis of 1,1,2-trifluoro-3,4-bis(trifluoromethyl)-1,4-

dihydronaphthalene (3) and 1,2-bistrifluoromethyl-3,4-difluoronaphthalene (2)<smiles>[Z]c1ccc(C(=C(C(F)=C(F)F)C(=C(C(=O)O)C(F)(F)F)C(F)(F)F)C(F)(F)F)cc1</smiles>

1<smiles>[Z]c1ccc2c(C(F)(F)F)c(C(F)(F)F)c(F)c(F)c2c1</smiles>

$2(46-59 \%)$<smiles>[Z]c1ccc2c(c1)C(F)(F)C(F)=C(C(F)(F)F)C2C(F)(F)F</smiles>

$3(14-26 \%)$

In the case of p-substituted perfluoro cis-1,2-dimethyl butadienyl benzenes

1a-d, photolysis of $\mathbf{1}$ leads to the unusual intramolecular cyclization and two principal products are obtained (Table 2). In these reactions, the 1,4dihydronaphthalene derivatives 3a-d were the minor photocyclization products. The predominant naphthalene derivatives 2a-d were obtained after photocyclization accompanied by photoelimination of HF.

Table 2. Photoreaction of $p$-substituted perfluoro-cis-1,2-dimethyl-butadienyl benzenes 1a-d.

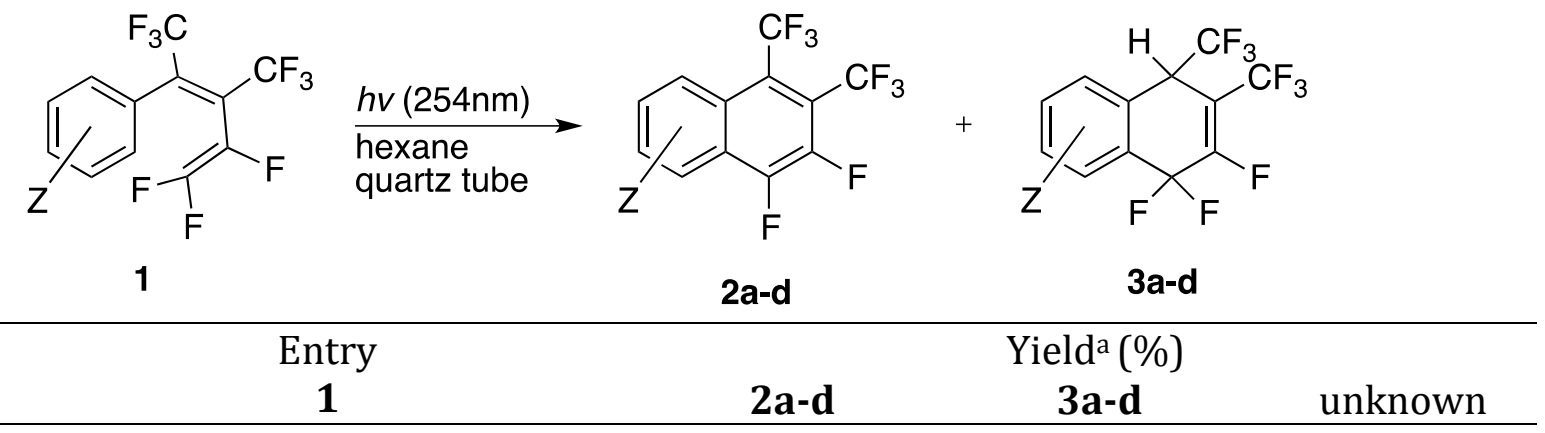




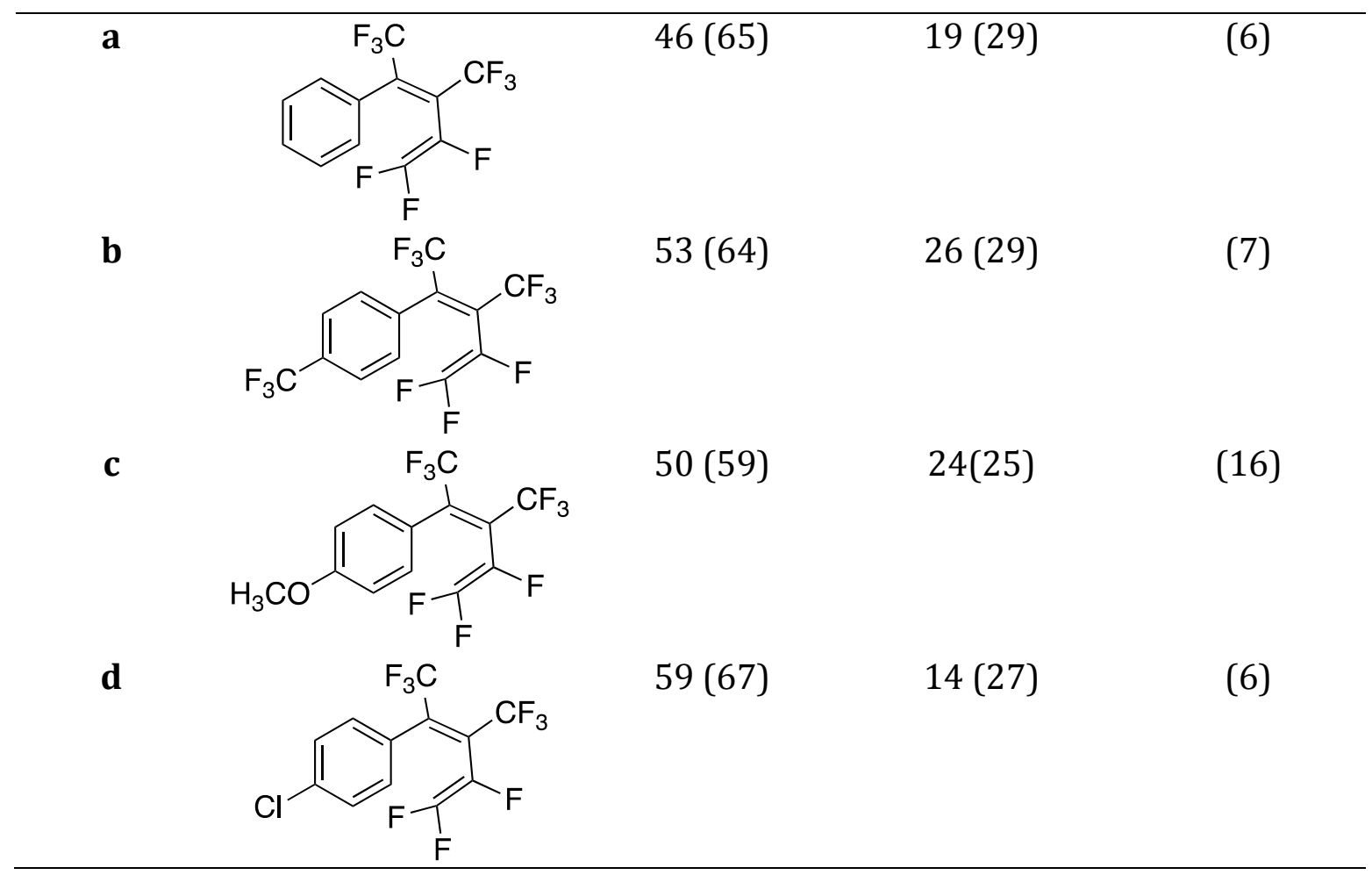

asolated yields (GC yields)

The formation of naphthalene derivatives $\mathbf{2 a - d}$ and 1,4-dihydronaphthalenes

3a-d were confirmed via GC-MS and NMR spectra analysis of isolated compounds

(Table 3 and 4 ).

Table 3. Selected NMR data of isolated $p$-naphthalene compounds $\mathbf{2 a - d}$.<smiles></smiles>

2a-d

\begin{tabular}{|c|c|c|c|c|c|c|c|c|}
\hline \multicolumn{2}{|l|}{ Entry } & \multicolumn{4}{|c|}{ ppm } & \multicolumn{3}{|c|}{$\mathrm{Hz}$} \\
\hline${ }^{19} \mathrm{~F}-\mathrm{NMR}$ & $\mathrm{Z}$ & $\mathrm{F}^{\mathrm{a}}(3 \mathrm{~F})$ & $\mathrm{F}^{\mathrm{b}}(3 \mathrm{~F})$ & $\mathrm{F}^{\mathrm{c}}(1 \mathrm{~F})$ & $\mathrm{F}^{\mathrm{d}}(1 \mathrm{~F})$ & $J_{a b}$ & $J_{\mathrm{bc}}$ & $J_{\mathrm{cd}}$ \\
\hline $2 a$ & $\mathrm{H}$ & $-51.4(q)$ & $-54.1(\mathrm{qd})$ & $-138.7(\mathrm{qd})$ & $-142.6(d)$ & 17 & 34 & 17 \\
\hline 2b & $\mathrm{CF}_{3}$ & $-51.4(q)$ & $-54.4(\mathrm{qd})$ & $-139.6(\mathrm{qd})$ & $-136.4(d)$ & 16 & 34 & 17 \\
\hline 2c & $\mathrm{OCH}_{3}$ & $-51.3(q)$ & $-53.7(q d)$ & $-142.2(\mathrm{qd})$ & $-140.3(d)$ & 16 & 32 & 16 \\
\hline 2d & $\mathrm{Cl}$ & $-51.5(q)$ & $-54.2(\mathrm{qd})$ & $-140.1(\mathrm{qd})$ & $-138.2(d)$ & 16 & 32 & 18 \\
\hline${ }^{1} \mathrm{H}-\mathrm{NMR}$ & $\mathrm{Z}$ & \multicolumn{4}{|c|}{ ppm } & \multicolumn{2}{|c|}{$\mathrm{Hz}$} & \\
\hline
\end{tabular}




\begin{tabular}{|c|c|c|c|c|c|c|c|c|c|}
\hline $2 a$ & $\mathrm{H}$ & \multicolumn{5}{|c|}{$7.7-8.2(4 \mathrm{H})$} & \multicolumn{3}{|c|}{ - } \\
\hline $2 b$ & $\mathrm{CF}_{3}$ & $7.9(1 \mathrm{H}, \mathrm{d})$ & $8.5(1 \mathrm{H}, \mathrm{d})$ & - & \multicolumn{2}{|c|}{$8.5(1 \mathrm{H}, \mathrm{s})$} & & 8 & \\
\hline $2 c$ & $\mathrm{OCH}_{3}$ & $7.1(1 \mathrm{H}, \mathrm{d})$ & $8.0(1 \mathrm{H}, \mathrm{d})$ & - & \multicolumn{2}{|c|}{$7.1(1 \mathrm{H}, \mathrm{s})$} & & 8 & \\
\hline 2d & $\mathrm{Cl}$ & $8.2(1 \mathrm{H}, \mathrm{d})$ & $8.5(1 \mathrm{H}, \mathrm{d})$ & - & & $1 \mathrm{H}, \mathrm{s})$ & & 9 & \\
\hline & \multicolumn{4}{|c|}{ ppm } & & & $\mathrm{Hz}$ & & \\
\hline${ }^{13} \mathrm{C}-\mathrm{NMR}$ & $\mathrm{Z}$ & $\mathrm{C}^{1}$ & $C^{3}$ & $\mathrm{C}^{4}$ & ${ }^{2} J_{1, \mathrm{~F}}$ & ${ }^{1} J_{3, \mathrm{~F}}$ & ${ }^{2} J_{3, \mathrm{~F}}$ & ${ }^{1} J_{4, \mathrm{~F}}$ & ${ }^{2} J_{4, \mathrm{~F}}$ \\
\hline $2 a$ & $\mathrm{H}$ & $123.7(q)$ & $144.3(\mathrm{dd})$ & $148.8(\mathrm{dd})$ & 34 & 258 & 14 & 255 & 14 \\
\hline $2 b$ & $\mathrm{CF}_{3}$ & $126.6(q)$ & $148.8(\mathrm{dd})$ & $144.7(\mathrm{dd})$ & 39 & 263 & 14 & 262 & 14 \\
\hline 2c & $\mathrm{OCH}_{3}$ & $123.3(q)$ & $144.3(\mathrm{dd})$ & $147.1(\mathrm{dd})$ & 36 & 257 & 14 & 256 & 14 \\
\hline 2d & $\mathrm{Cl}$ & $124.1(\mathrm{q})$ & $147.3(\mathrm{dd})$ & $144.6(\mathrm{dd})$ & 32 & 261 & 14 & 259 & 14 \\
\hline
\end{tabular}

Table 4. Selected NMR data of isolated 1,4-dihydronaphthalene compounds 3a-d.<smiles>[Z]c1ccc2c(c1)C(F)(F)C(F)=C(C(F)(F)F)C2([2H])C(F)(F)F</smiles>

3a-d

\begin{tabular}{|c|c|c|c|c|c|c|c|c|c|c|c|c|}
\hline \multirow{2}{*}{$\begin{array}{c}\text { Entry } \\
19 \mathrm{~F}-\mathrm{NMR}\end{array}$} & \multirow[b]{2}{*}{$\mathrm{Z}$} & \multirow[b]{2}{*}{$\mathrm{F}^{\mathrm{a}}(3 \mathrm{~F})$} & \multirow{2}{*}{\multicolumn{2}{|c|}{$\mathrm{F}^{\mathrm{b}}(3 \mathrm{~F})$}} & \multicolumn{4}{|c|}{$\mathrm{ppm}$} & & & \multicolumn{2}{|c|}{$\mathrm{Hz}$} \\
\hline & & & & & \multicolumn{2}{|c|}{$\mathrm{Fc}^{\mathrm{c}}(1 \mathrm{~F})$} & $\mathrm{F}^{\mathrm{d}}(1 \mathrm{~F})$ & & & & $J_{a, H 5}$ & $J_{\mathrm{cd}}$ \\
\hline $3 a$ & $\mathrm{H}$ & $-60.7(d)$ & \multicolumn{2}{|r|}{$-69.6(s)$} & \multicolumn{2}{|c|}{$-114.9(\mathrm{~m})$} & \multicolumn{2}{|c|}{$-85.3(d d)$} & \multicolumn{2}{|c|}{$-101.3(\mathrm{dd})$} & 7 & 304 \\
\hline $3 \mathbf{b}$ & $\mathrm{CF}_{3}$ & $-60.8(d)$ & \multicolumn{2}{|r|}{$-69.3(\mathrm{~s})$} & \multicolumn{2}{|c|}{$-114.2(\mathrm{~m})$} & $-85.5(d d)$ & \multicolumn{3}{|c|}{$-101.3(\mathrm{dd})$} & 7 & 306 \\
\hline $3 c$ & $\mathrm{OCH}_{3}$ & $-60.7(d)$ & \multicolumn{2}{|r|}{$-69.9(\mathrm{~s})$} & \multicolumn{2}{|c|}{$-115.6(\mathrm{~m})$} & \multicolumn{2}{|c|}{$-85.4(d d)$} & \multicolumn{2}{|c|}{$-1013(\mathrm{dd})$} & 6 & 304 \\
\hline \multirow[t]{2}{*}{$3 d$} & $\mathrm{Cl}$ & $-60.8(d)$ & \multicolumn{2}{|r|}{$-69.6(\mathrm{~s})$} & \multirow{2}{*}{\multicolumn{2}{|c|}{$\frac{-114.7(\mathrm{~m})}{\mathrm{ppm}}$}} & & \multicolumn{2}{|c|}{$-101.3(\mathrm{dd})$} & 6 & 304 \\
\hline & & & & & & & $-85.5(d d)$ & & & & \multicolumn{2}{|c|}{$\mathrm{Hz}$} \\
\hline${ }^{1} \mathrm{H}-\mathrm{NMR}$ & $\mathrm{Z}$ & $\mathrm{H}^{1}$ & & & $\mathrm{y}^{2}$ & $\mathrm{H}^{3}$ & $\mathrm{H}^{4}$ & & $\mathrm{H}^{5}$ & & $J_{1,2}$ & $J_{1, F a}$ \\
\hline $3 a$ & $\mathrm{H}$ & \multicolumn{5}{|c|}{$7.9-7.5(4 \mathrm{H})$} & & \multicolumn{3}{|c|}{$4.6(1 \mathrm{H}, \mathrm{q})$} & - & 7 \\
\hline $3 \mathbf{b}$ & $\mathrm{CF}_{3}$ & \multicolumn{3}{|c|}{$7.7(1 \mathrm{H}, \mathrm{d})$} & $(H, d)$ & - & $8.1(1 \mathrm{H}, \mathrm{s})$ & \multicolumn{3}{|c|}{$4.6(1 \mathrm{H}, \mathrm{q})$} & 8 & 7 \\
\hline $3 c$ & $\mathrm{OCH}_{3}$ & $7.1(1 \mathrm{H}$ & & 7.4( & $1 \mathrm{H}, \mathrm{d})$ & - & $7.3(1 \mathrm{H}, \mathrm{s})$ & & $4.5(1 \mathrm{H}$ & & 9 & 6 \\
\hline 3d & $\mathrm{Cl}$ & $7.4(1 \mathrm{H}$ & & 7.61 & $1 \mathrm{H}, \mathrm{d})$ & - & $7.8(1 \mathrm{H}, \mathrm{s})$ & & $4.5(1 \mathrm{H}$ & & 8 & 6 \\
\hline & & & & & ppm & & & & & & $\mathrm{Hz}$ & \\
\hline${ }^{13} \mathrm{C}-\mathrm{NMR}$ & $\mathrm{Z}$ & $\mathrm{C}^{1}$ & & $\mathrm{C}^{2}$ & $\mathrm{C}^{3}$ & & $\mathrm{C}^{4}$ & & $\mathrm{C}^{5}$ & ${ }^{2} J_{1, \mathrm{~F}}$ & $1_{3, \mathrm{~F}}$ & ${ }^{1} J_{4, \mathrm{~F}}$ \\
\hline $\mathbf{3 a}$ & $\mathrm{H}$ & $43.7(q)$ & & $1.1(\mathrm{~m})$ & $156.7(d$ & & $109.9(\mathrm{td})$ & & $1.0(\mathrm{t})$ & 31 & 294 & 236 \\
\hline $3 \mathbf{b}$ & $\mathrm{CF}_{3}$ & 43.8(q) & & $1.1(\mathrm{~m})$ & $156.4(\mathrm{~d}$ & & $109.3(\mathrm{td})$ & & $2.1(\mathrm{t})$ & 31 & 294 & 235 \\
\hline $3 c$ & $\mathrm{OCH}_{3}$ & $43.2(q)$ & & $1.1(\mathrm{~m})$ & $156.7(\mathrm{~d}$ & & $109.7(\mathrm{td})$ & & $2.2(\mathrm{t})$ & 31 & 294 & 239 \\
\hline $3 d$ & $\mathrm{Cl}$ & $43.7(\mathrm{q})$ & 11 & $1.1(\mathrm{~m})$ & 156.7 d & & $109.4(\mathrm{td})$ & & $3.4(\mathrm{t})$ & 31 & 294 & 239 \\
\hline
\end{tabular}

The ${ }^{19} \mathrm{~F}-\mathrm{NMR}$ spectrum of the naphthalene derivatives showed the resonance of the two fluorines and two trifluoromethyl groups instead of the three vinyl fluorines and two trifluoromethyl groups which were observed in the staring compounds 1a-d. The coupling patterns of the two aryl fluorines (doublet, $J=16-18$ $\mathrm{Hz}$ and quartet-doublet, $J=32-34,16-18 \mathrm{~Hz}$ ) and the two trifluoromethyl groups 
(quartet, $J=16-17 \mathrm{~Hz}$, and doublet-quartet, $J=32-34,16-17 \mathrm{~Hz}$ ) supported these naphthalene structures (Table 3).

In contrast, ${ }^{19} \mathrm{~F}-\mathrm{NMR}$ spectra of 1,4-dihydronaphthalene derivatives 3a-d exhibited a fluorine, difluoromethylene, and two trifluoromethyl groups (Table 4). Splitting of methylene $\mathrm{H} 5$ into a quartet $\left(3_{\mathrm{H} 5, \mathrm{~F}}=6-7 \mathrm{~Hz}\right)$ in the ${ }^{1} \mathrm{H}-\mathrm{NMR}$ spectrum and splitting of $\mathrm{C} 1\left({ }^{2} J_{\mathrm{C} 1, \mathrm{~F}}=31 \mathrm{~Hz}\right)$ in the ${ }^{13} \mathrm{C}-\mathrm{NMR}$ spectrum supports the assigned structures. The existence of three (3b-d) or four (3a) aromatic protons, excluding H5 (since no structures could be written without breaking aromaticity), provides further support.

To our knowledge, only two examples of photocyclizations which are accompanied by HF photoelimination have been reported. Although the reaction mechanism are not clearly understood, Fokin and coworkers [10] found that Nmethyl-2,3,4,5-tetrafluoro diphenyl amine was converted to 2,3,4-trifluoro-9-methyl carbazole with HF photoelimination. Mallory [11] also found that 1-

fluoro[5]helicenes proceed through HF photoelimination in their conversion to benzo[ghi]perylene. An NMR study of this compound was independently carried out by Gorsane [12].

The treatment of isolated 3a with 1,4-diazabicyclo[2.2.2] octane base (DABCO), resulted in the naphthalene derivative $\mathbf{2 a}$ (Scheme 3). Photolysis of 3a, however, does not afford 2a, implicating an intermediate other than $\mathbf{3 a}$ as the photochemical precursor to $\mathbf{2 a}$.

Scheme 3. HF elimination of 3a to $\mathbf{2 a}$ with use of DABCO 
<smiles>FC1=C(C(F)(F)F)C(C(F)(F)F)c2ccccc2C1(F)F</smiles>

3a

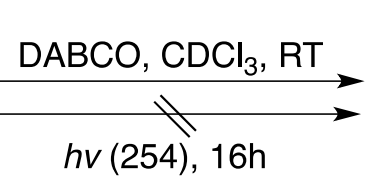

$h v(254), 16 \mathrm{~h}$<smiles>Fc1c(C(F)(F)F)c(C(F)(F)F)c2ccccc2c1F</smiles>

2a

The photolysis of 1,4-bis(perfluoro cis-1,2-dimethyl-1,3-butadienyl) benzene $\mathbf{1 f}$ leads to multiple products. Among them, two major products, anthracene (7) and phenanthrene (8) could be isolated (Scheme 4). In this reaction, phenanthrene 8 is the favored product, although for reasons unclear. In conjunction with various analytical data (e.g. NMR), X-ray crystal analysis data (Fig. 1) also assisted in the assignment of chemical structures. From X-ray analysis, phenanthrene derivative 8 has a twisted structure (twisted angle of 8 is $27^{\circ}$ between ring A and C), while anthracene derivative 7 has a planar structure [13].

Scheme 4. Photolysis of $\mathbf{1 f}$ to lead $\mathbf{7}$ and $\mathbf{8 .}$

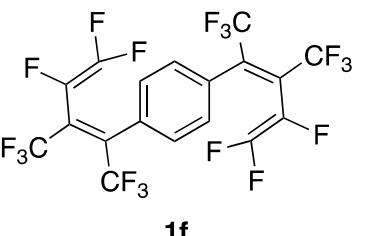

$1 \mathrm{f}$ $h v(254 \mathrm{~nm})$<smiles>Fc1c(F)c(C(F)(F)F)c2cc3c(F)c(F)c(C(F)(F)F)c(C(F)(F)F)c3cc2c1F</smiles><smiles>Fc1cc2ccc3c(C(F)(F)F)c(C(F)(F)F)c(F)c(C(F)(F)F)c3c2c(C(F)(F)F)c1F</smiles>

Figure 1. Crystal Sturture of a) 7 and b) 8 . 

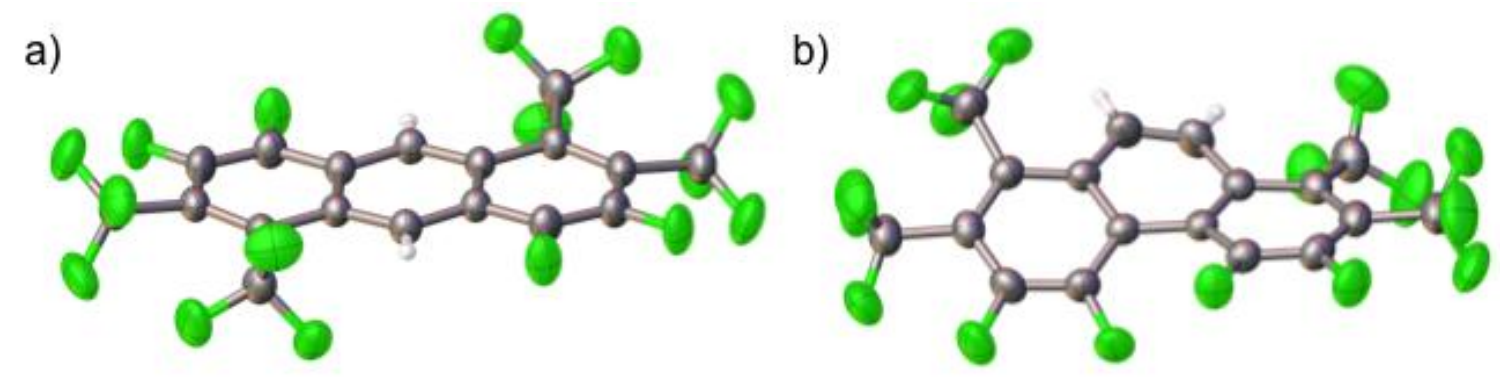

Photolysis of $m$-methyl substituted benzene 1e was carried out under similar conditions and generates four major products (Scheme 5). The obtained products could not be completely separated due to extremely close boiling points and similar TLC behavior. However, the naphthalene derivatives and 1,4-dihydronaphthalene derivatives could be separated as mixtures of $\mathbf{2 e} / \mathbf{2} \mathbf{e}^{\prime}$ and $\mathbf{3 e} / \mathbf{3} \mathbf{e}^{\prime}$.

Scheme 5. Photoreaction of $m-\mathrm{CH}_{3}$ substituted 1e.
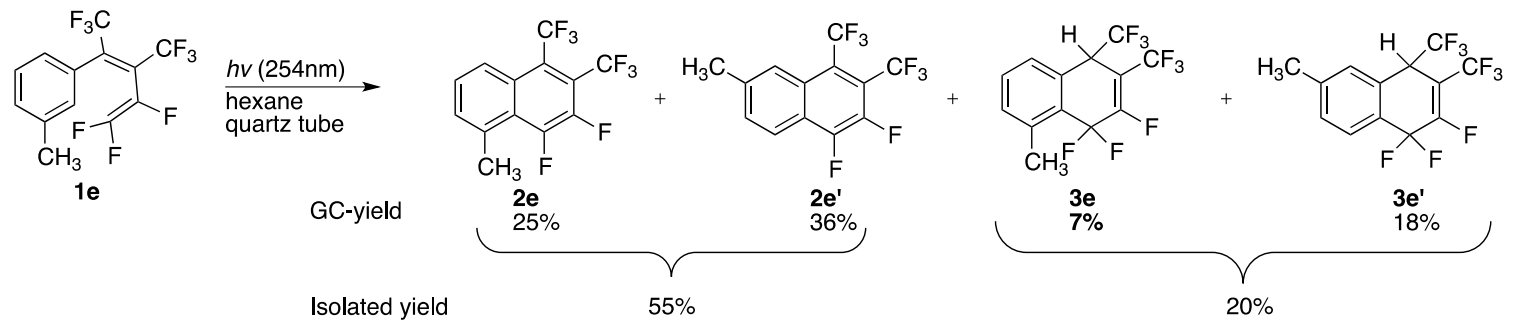

From the NMR spectrum and GC yields, the mixture of naphthalene derivatives $\mathbf{2 e} / \mathbf{2} \mathbf{e}^{\prime}$ consisted of $40 \% \mathbf{2 e}$ and $60 \% \mathbf{2} \mathbf{e}^{\prime}$. Having similar ${ }^{19} \mathrm{~F}-\mathrm{NMR}$ spectra (Table 5), compounds $\mathbf{2 e}$ and $\mathbf{2} \mathbf{e}^{\prime}$ were differentiated on the basis of the respective $\mathrm{H} 4$ coupling patterns in the ${ }^{1} \mathrm{H}-\mathrm{NMR}$. For the methyl $\mathrm{H} 4$ protons, compound $\mathbf{2 e}$ ' exhibited a singlet, while the $\mathbf{2 e}$ methyl $4 \mathrm{H}$ protons were split into a doublet by the adjacent $\mathrm{Fd}\left(\mathrm{J}_{\mathrm{H} 4-\mathrm{Fd}}=8 \mathrm{~Hz}\right)$. 
Table 5. NMR data of m-naphthalene mixtures $\mathbf{2 e}$ and $\mathbf{2} \mathbf{e}^{\prime}$.<smiles>Cc1cccc2c(C(F)(F)F)c(C(F)(F)F)c(F)c(F)c12</smiles>

$2 e$<smiles>Cc1cccc2c(C(F)(F)F)c(C(F)(F)F)c(F)c(F)c12</smiles>

$2 e^{\prime}$

\begin{tabular}{|c|c|c|c|c|c|c|}
\hline Entry & \multicolumn{4}{|c|}{ ppm } & \multicolumn{2}{|c|}{$\mathrm{Hz}$} \\
\hline${ }^{19} \mathrm{~F}-\mathrm{NMR}$ & $\mathrm{Fa}(3 \mathrm{~F})$ & $\mathrm{Fb}^{\mathrm{b}}(3 \mathrm{~F})$ & $\mathrm{Fc}^{\mathrm{c}}(1 \mathrm{~F})$ & $\mathrm{F}^{\mathrm{d}}(1 \mathrm{~F})$ & $J_{\mathrm{bc}}$ & $J_{\mathrm{cd}}$ \\
\hline $2 \mathbf{e}$ & $-51.4(\mathrm{~m})$ & $-54.2(\mathrm{~m})$ & $-143.0(q d)$ & $-129.7(\mathrm{bs})$ & 34 & 14 \\
\hline $2 e^{\prime}$ & $-51.4(\mathrm{~m})$ & $-54.0(\mathrm{~m})$ & -144.1 (qd) & $-139.2(\mathrm{~d})$ & 34 & 18 \\
\hline & \multicolumn{4}{|c|}{ ppm } & \multicolumn{2}{|c|}{$\mathrm{Hz}$} \\
\hline${ }^{1} \mathrm{H}-\mathrm{NMR}$ & $\mathrm{H}^{1-3}$ & \multicolumn{3}{|c|}{$\mathrm{H}^{4}$} & \multicolumn{2}{|c|}{$J_{4, F d}$} \\
\hline $2 \mathrm{e}$ & $7.4-7.9(3 \mathrm{H}, \mathrm{m})$ & \multicolumn{3}{|c|}{$2.8(\mathrm{~d})$} & \multicolumn{2}{|c|}{8} \\
\hline $2 e^{\prime}$ & 7.4-7.9 $(3 \mathrm{H}, \mathrm{m})$ & \multicolumn{3}{|c|}{$2.8(d)$} & \multicolumn{2}{|c|}{0} \\
\hline
\end{tabular}

Lack of diagnostic difference in the NMR spectra prevented the direct assignment of either $\mathbf{3 e}$ or $\mathbf{3} \mathbf{e}^{\prime}$ as the major component in the observed $\mathbf{3 / 7}$ ratio. However, a mixture of the $\mathbf{3 e} / \mathbf{3} \mathbf{e}^{\prime}$ was converted to a $\mathbf{3} / \mathbf{7}$ mixture of the $\mathbf{2 e} / \mathbf{2} \mathbf{e}^{\prime}$ by treatment with diazabicycloundecene (DBU) in $\mathrm{CDCl}_{3}$ (Scheme 6). The ${ }^{19} \mathrm{~F}-\mathrm{NMR}$ spectrum of the reaction mixture was consistent with the structures $\mathbf{2 e}$ and $\mathbf{2 e}$ '. Thus, the conversion of 1,4-dihydronaphthalene derivatives to the naphthalene derivatives provided the structural confirmation and facilitated determination of the $\mathbf{3 e} / \mathbf{3} \mathbf{e}^{\prime}$ ratio (Table 6).

Table 6. NMR data of 1,4-dihydronaphthalene mixtures $\mathbf{3 e}$ and $\mathbf{3 e}$ '.<smiles>Cc1ccc2c(c1)C(F)(F)C(F)=C(C(F)(F)F)C2C(F)(F)F</smiles>

3 e<smiles>Cc1ccc2c(c1)C(C(F)(F)F)C(C(F)(F)F)=C(F)C2(F)F</smiles>

$3 e^{\prime}$

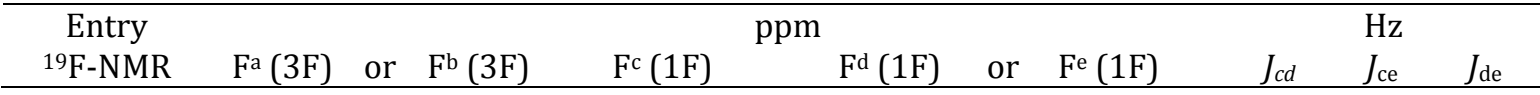




\begin{tabular}{|c|c|c|c|c|c|c|c|c|}
\hline $3 \mathbf{e}$ & $-60.9(\mathrm{~m})$ & $-69.9(\mathrm{~s})$ & $-115.4(\mathrm{~m})$ & $-93.8(d d)$ & $-95.8(\mathrm{dd})$ & 22 & 14 & 309 \\
\hline $3 e^{\prime}$ & $-60.9(\mathrm{~m})$ & $-69.6(\mathrm{~s})$ & $-115.1(\mathrm{~m})$ & $-84.9(\mathrm{dd})$ & $-101.1(\mathrm{dd})$ & 23 & 13 & 303 \\
\hline & \multicolumn{6}{|c|}{ ppm } & \multicolumn{2}{|c|}{$\mathrm{Hz}$} \\
\hline${ }^{1} \mathrm{H}-\mathrm{NMR}$ & \multicolumn{2}{|c|}{$\mathrm{H}^{1-3}$} & \multicolumn{2}{|c|}{$\mathrm{H}^{4}$} & \multicolumn{2}{|c|}{$\mathrm{H}^{5}$} & & \\
\hline $\mathbf{3 e}$ & \multirow{2}{*}{\multicolumn{2}{|c|}{$\begin{array}{l}7.3-7.7(3 \mathrm{H}, \mathrm{m}) \\
7.3-7.7(3 \mathrm{H}, \mathrm{m})\end{array}$}} & \multirow{2}{*}{\multicolumn{2}{|c|}{$\begin{array}{c}2.6(1 \mathrm{H}, \mathrm{m}) \\
2.4(1 \mathrm{H}, \mathrm{s})\end{array}$}} & \multirow{2}{*}{\multicolumn{2}{|c|}{$\begin{array}{l}4.5(1 \mathrm{H}, \mathrm{m}) \\
4.4(1 \mathrm{H}, \mathrm{m})\end{array}$}} & & \\
\hline $3 \mathbf{e}^{\prime}$ & & & & & & & & \\
\hline
\end{tabular}

Scheme 6. Conversion of the mixture $3 \mathbf{e} / 3 \mathbf{e}^{\prime}$ into $2 \mathbf{e} / \mathbf{2} \mathbf{e}^{\prime}$.

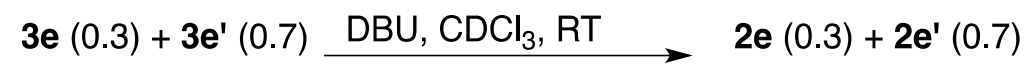

To probe the photoreaction mechanism, we initially hypothesized the involvement of electrophilic attack on the benzene ring and that this step might be slowest and rate determining. As a consequence, we expect that reaction rates would be sensitive to the nature of the substituent on the benzene ring. That is, electron-donating groups should enhance reaction rate through stabilization of the intermediate cation while electron-withdrawing groups slow the reaction rate through destabilization. Competitive photoreaction studies were carried out between several perfluoro cis-1,2-dimethyl butadienyl benzenes 1. Direct competition between non-substituted benzene $\mathbf{1 a}$ and $m$-methyl-substituted benzene 1e, however resulted in little difference in reaction rate, suggesting that the methyl inductive effect contributed insignificantly to the reaction rate. Similarly, competition between $p$-trifluoromethyl-substituted benzene $\mathbf{1 b}$ and nonsubstituted 1 a resulted in no significant decrease in reaction rate. The failure of both $p$-trifluoromethyl $\mathbf{1 b}$ and $m$-methyl $\mathbf{1 e}$ to significantly influence reaction rate was inconsistent with a mechanism in which electrophilic attack was rate 
determining. Additional detailed studies are required to elucidate other mechanistic pathways (i.e. radical).

Figure 2. Competitive photolysis of $\mathbf{1 a}$ (dash, ---) and $\mathbf{1 e}\left(\mathrm{m}-\mathrm{CH}_{3}\right.$, solid, -).

Consumption of $\mathbf{1 a} \& \mathbf{1 e}$

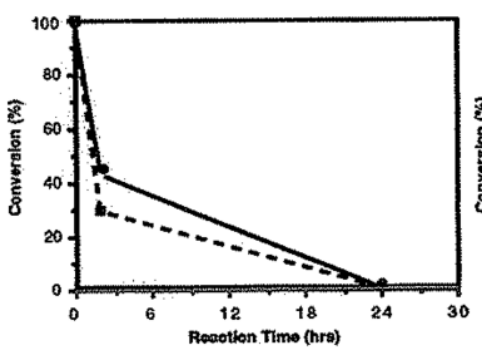

Formation of 1,4dihydronaphthalenes $\mathbf{3 a} \& \mathbf{3 e} / \mathbf{3} \mathbf{e}^{\prime}$

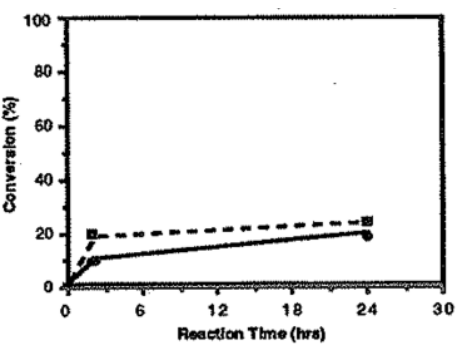

Formation of Naphthalenes 3a \&

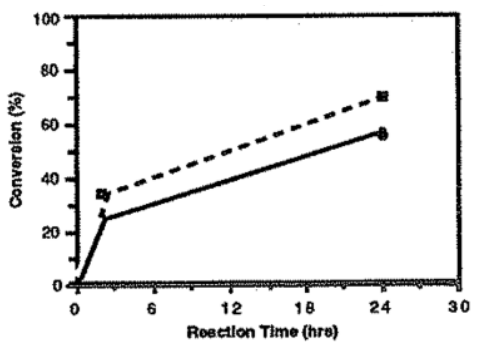

Figure 3. Competitive photolysis of $\mathbf{1 a}$ (dash, ---) and $\mathbf{1 b}\left(\mathrm{p}-\mathrm{CF}_{3}\right.$, solid, -).

Consumption of $\mathbf{1 a} \& \mathbf{1 b}$

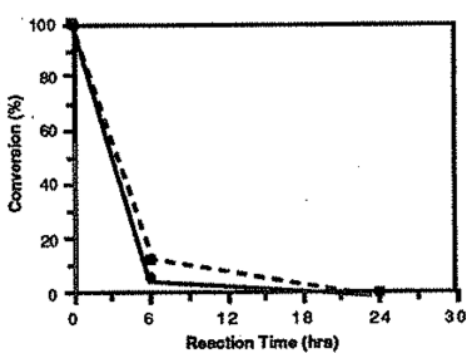

Formation of 1,4dihydronaphthalenes $\mathbf{3 a} \& \mathbf{3 b}$

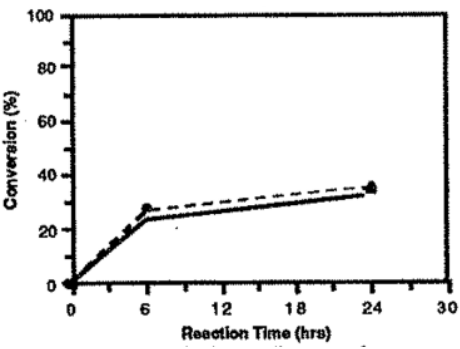

Formation of Naphthalenes $\mathbf{3 a} \& \mathbf{3 b}$

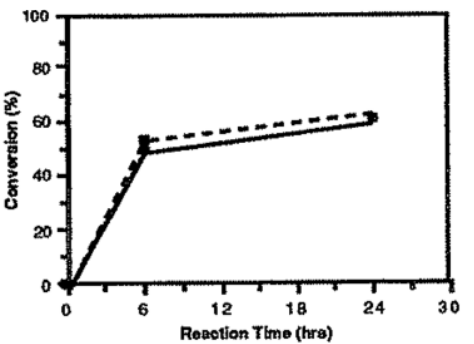

\section{Experimental}

\subsection{General experimental procedures}

Routine ${ }^{19}$ F-NMR spectra were recorded on a JEOL FX90Q Spectrometer

(83.81 MHz) and high-resolution data was obtained on a Bruker AC-300

Spectrometer (282.41 MHz). Chemical shifts have been reported in ppm relative to internal $\mathrm{CFCl}_{3}$. Spectra of reaction mixtures were obtained in the ${ }^{7} \mathrm{Li}$ external lock 
mode. Quantitative determinations were carried out by integration relative to internal benzotrifluoride. Routine ${ }^{1} \mathrm{H}-\mathrm{NMR}(300.17 \mathrm{MHz})$ spectra and highresolution data were generally obtained on a Bruker AC-300 Spectrometer. Unless noted otherwise, $\mathrm{CDCl}_{3}$ was used as the NMR lock solvent. Chemical shifts have been reported in ppm relative to internal TMS. High resolution $\left\{{ }^{1} \mathrm{H}\right\}{ }^{13} \mathrm{C}$ NMR spectra were recorded on a Bruker AC-300 Spectrometer (75.48 MHz). Chemical shifts have been reported in ppm relative to internal TMS. High-resolution mass spectra were obtained by the University of Iowa High Resolution Mass Spectrometry Facility. Photolysis experiments were preformed in a Rayonet Reactor with $254 \mathrm{~nm}$ bulbs. Analytical GLPC were performed on a Hewlett-Packard Model 5890 equipped with a thermal conductivity detector and 3393A integrator. The column was packed with 5\% OV-101 on chromo- sorb P. UV measurements (hexane) were made with a Hewlett-Packard 8452A diode array spectrophotometer. All boiling points were determined during fractional distillation using a partial immersion thermometer and are uncorrected. 4 Å molecular sieves (Fisher) were activated by heating under vacuum $\left(300^{\circ} \mathrm{C}\right.$ at $0.5 \mathrm{~mm} \mathrm{Hg}$ ) overnight. DMF was dried overnight over $\mathrm{CaH}_{2}$ and then distilled at reduced pressure. Silica gel was purchased from EM Science (Silica Gel 60, particle sized 0.063-0.200 $\mu \mathrm{m}, 70-230 \mathrm{Mesh}, \mathrm{ASTM})$. CuBr was purified by the method reported by Morken [14]. Hexafluorobutyne can be prepared by the zinc dehalogenation of $\mathrm{CF}_{3} \mathrm{CCl}_{2} \mathrm{CCl}_{2} \mathrm{CF}_{3}$ or purchased from PCR Specialty Chemicals. Bromotrifluoroethylene (Halocarbon), DBU, DABCO, and substituted aryl iodides were obtained from commercial sources and used without further purification. 


\subsection{Activation of Zinc Powder}

Zinc powder (70 g) was suspended in $200 \mathrm{~mL}$ of water and $3 \mathrm{~mL}$ of conc. $\mathrm{HCl}$ was added dropwise. After stirring for $15 \mathrm{~min}$, the precipitate was washed with water $(3 \times 500 \mathrm{~mL})$. The zinc powder was then dried under vacuum for $3 \mathrm{~h}$.

\subsection{Preparation of $\mathrm{CF}_{2}=\mathrm{CFCu}(\mathbf{5})$,}

An oven-dried one-liter 3-necked flask, equipped with a dry ice/IPA condenser, $\mathrm{N}_{2}$ inlet, septum port, and magnetic stirring bar, was charged with activated Zn powder (19 g, $290 \mathrm{mmol})$ and dry DMF (300 mL). Then trifluorobromoethylene $(16 \mathrm{~g}, 100 \mathrm{mmol})$ was then slowly added into this solution. The resultant mixture was gently heated with a heat gun until an exothermic reaction $\left(50-60^{\circ} \mathrm{C}\right)$ occurred. Additional trifluorobromoethylene $(32 \mathrm{~g}, 200 \mathrm{mmol})$ was then condensed into the solution, maintaining the internal temperature for an additional $3 \mathrm{~h}$, and then unreacted olefin was removed by vacuum. The NMR yield of $\mathrm{CF}_{2}=\mathrm{CFZnBr}$ was $62 \%$. A $1000 \mathrm{~mL}$ two-necked flask, equipped with dry ice-IPA condenser, $\mathrm{N}_{2}$ inlet, and septum port was charged with $\mathrm{CuBr}$ (29.4 g, $\left.192 \mathrm{mmol}\right)$. To this flask, the $\mathrm{CF}_{2}=\mathrm{CFZnBr} / \mathrm{DMF}$ solution $(0.62 \mathrm{M}, 300 \mathrm{~mL}, 186 \mathrm{mmol})$ was transferred by syringe. The reaction mixture was stirred for $20 \mathrm{~min}$ at room temperature and then check by ${ }^{19} \mathrm{~F}$ NMR to verify formation of the copper reagent [15]. $\mathrm{CF}_{2}=\mathrm{CFZnBr}:{ }^{19} \mathrm{~F}$ NMR (83.9 MHz, JEOL FX-90Q, DMF) -94.7 ppm (dd, 1F, cis$\left.\mathrm{CF}=\mathrm{CF}_{2}, \mathrm{Fa}\right),-128.9 \mathrm{ppm}\left(\mathrm{dd}, 1 \mathrm{~F}\right.$, trans $\left.-\mathrm{CF}=\mathrm{CF}_{2}, \mathrm{Fb}\right),-193.3 \mathrm{ppm}\left(\mathrm{dd}, 1 \mathrm{~F}, \mathrm{CF}=\mathrm{CF}_{2}, \mathrm{Fc}\right)$, $\left.U_{\mathrm{ab}}=93 \mathrm{~Hz}, J_{\mathrm{ac}}=34 \mathrm{~Hz}, J_{\mathrm{bc}}=105 \mathrm{~Hz}\right) . \mathrm{CF} 2=\mathrm{CFCu}:{ }^{19} \mathrm{~F}$ NMR $(83.9 \mathrm{MHz}, \mathrm{JEOL} F X-90 \mathrm{Q}$,

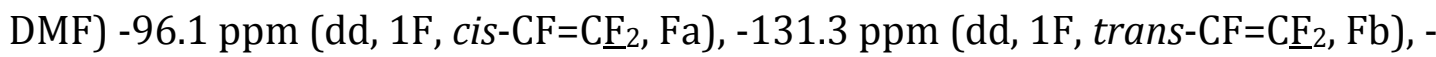
$182.8 \mathrm{ppm}(\mathrm{dd}, 1 \mathrm{~F}, \mathrm{CF}=\mathrm{CF} 2, \mathrm{Fc}),\left(U_{\mathrm{ab}}=100 \mathrm{~Hz}, J_{\mathrm{ac}}=32 \mathrm{~Hz}, J_{\mathrm{bc}}=100 \mathrm{~Hz}\right)$. 
3.4 Preparation of $\mathrm{CF}_{2}=\mathrm{CF}-\mathrm{C}\left(\mathrm{CF}_{3}\right)=C\left(\mathrm{CCF}_{3}\right) \mathrm{Cu}(\mathbf{6})$,

F-2-butyne (33.0 g, $206 \mathrm{mmol}$ ) was slowly condensed into the above copper DMF solution of 5 at $0{ }^{\circ} \mathrm{C}$. The reaction mixture was stirred for $3 \mathrm{~h}$ at room temperature. The NMR yield (based on $\mathrm{CF}_{2}=\mathrm{CFZnBr}$ ) was quantitative. ${ }^{19} \mathrm{~F} \mathrm{NMR}$

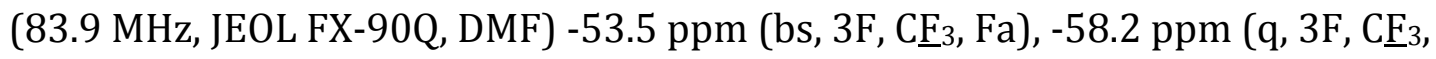

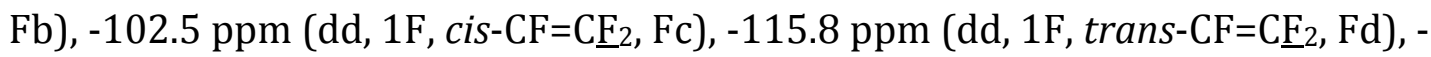
$\left.158.5 \mathrm{ppm}\left(\mathrm{dd}, 1 \mathrm{~F}, \mathrm{CF}=\mathrm{CF}_{2}, \mathrm{Fe}\right), \mathrm{U}_{\mathrm{cd}}=73 \mathrm{~Hz}, J_{\mathrm{ce}}=28 \mathrm{~Hz}, J_{\mathrm{de}}=117 \mathrm{~Hz}\right)$.

\subsection{Preparation of Z-1,1,2,5,5,5-hexafluoro-4-aryl-3-trifluoromethyl-1,3-} pentadiene derivatives (1a-f).

A $250 \mathrm{~mL}$ two-necked flask, equipped with magnetic stirrer and septum port was charged with copper compound 6 (42 mL, $26 \mathrm{mmol}$ ). Then aryl iodine (18 mmol) was added at once and the solution was heated with an oil bath to $70-80^{\circ} \mathrm{C}$ and stirred for $16 \mathrm{~h}$ under $\mathrm{N}_{2}$. The cooled reaction mixture was poured into water $(50 \mathrm{~mL})$ and ether $(100 \mathrm{~mL})$. The ether layer was washed with water $(2 \times 50 \mathrm{~mL})$ and dried over anhydrous $\mathrm{MgSO}_{4}$. After filtration, a rotary evaporator removed the solvent. The crude mixture was purified via silica gel (70-230 mesh) chromatography (hexane). Yields are also shown in Table 1.

1a: Yield $5.8 \mathrm{~g}(76 \%)$; GLPC purity 97\%; ${ }^{19} \mathrm{~F}$ NMR $\left(\mathrm{CDCl}_{3}\right)-59.6 \mathrm{ppm}\left(\mathrm{q}, 3 \mathrm{~F},-\mathrm{CF}_{3}, \mathrm{Fa}\right)$, $-60.3 \mathrm{ppm}\left(\mathrm{q}, 3 \mathrm{~F},-\mathrm{CF}_{3}, \mathrm{Fb}\right),-99.1 \mathrm{ppm}\left(\mathrm{dd}, 1 \mathrm{~F}, c i s-\mathrm{CF}=\mathrm{CF}_{2}, \mathrm{Fc}\right.$ ), $-111.9 \mathrm{ppm}(\mathrm{dd}, 1 \mathrm{~F}$, trans $\left.-\mathrm{CF}=\mathrm{CF}_{2}, \mathrm{Fd}\right),-165.4 \mathrm{ppm}\left(\mathrm{dd}, 1 \mathrm{~F},-\mathrm{CF}=\mathrm{CF}_{2}, \mathrm{Fe}\right),\left(\mathrm{Uab}_{\mathrm{ab}}=13 \mathrm{~Hz}, J_{\mathrm{cd}}=61 \mathrm{~Hz}, J_{\mathrm{ce}}=32\right.$ $\left.\mathrm{Hz}, J_{\mathrm{de}}=117 \mathrm{~Hz}\right) ;{ }^{1} \mathrm{H}$ NMR $\left(\mathrm{CDCl}_{3}\right) 7.2-7.4 \mathrm{ppm}(\mathrm{m}, 5 \mathrm{H}) ;{ }^{13} \mathrm{C}\left\{{ }^{1} \mathrm{H}\right\} \mathrm{NMR}\left(\mathrm{CDCl}_{3}\right) 153.2$ ppm (ddd, $\left.-\mathrm{CF}=\underline{C F}_{2},{ }^{1} J_{\mathrm{CF}}=294 \mathrm{~Hz}, 280 \mathrm{~Hz},{ }^{2} J_{\mathrm{CF}}=46 \mathrm{~Hz}\right), 148.3 \mathrm{ppm}\left(\mathrm{q}, \phi-\underline{\mathrm{C}}\left(\mathrm{CF}_{3}\right)=\mathrm{C}\right.$, ${ }^{2} J_{\mathrm{CF}}=32 \mathrm{~Hz}$ ), $131.9 \mathrm{ppm}(\mathrm{s}), 130.8 \mathrm{ppm}(\mathrm{s}), 129.1 \mathrm{ppm}(\mathrm{s}), 128.1 \mathrm{ppm}(\mathrm{s}), 126.2 \mathrm{ppm}$ $\left(\mathrm{m},-\mathrm{C}\left(\mathrm{CF}_{3}\right)=\underline{\mathrm{C}}\left(\mathrm{CF}_{3}\right)-\mathrm{CF}\right), 122.4 \mathrm{ppm}\left(\mathrm{ddd},-\underline{\mathrm{CF}}=\mathrm{CF}_{2},{ }^{1} J_{\mathrm{CF}}=236 \mathrm{~Hz},{ }^{2} J_{\mathrm{CF}}=54 \mathrm{~Hz}, 24 \mathrm{~Hz}\right)$, $121.1 \mathrm{ppm}\left(\mathrm{q},-\underline{C F}_{3},{ }^{1} J_{\mathrm{CF}}=275 \mathrm{~Hz}\right), 120.9 \mathrm{ppm}\left(\mathrm{q},-\underline{C F}_{3},{ }^{1} J_{\mathrm{CF}}=275 \mathrm{~Hz}\right) ; \mathrm{GC}-\mathrm{MS} 320\left(\mathrm{M}^{+}\right.$, 
13), 300 (M+-HF, 17), 281 (13), 251 (100), 231 (42), 201 (83), 182 (74), 151 (37); HRMS ( $\mathrm{C}_{12} \mathrm{H}_{5} \mathrm{~F}_{9}$, calculated: 320.0248 , observed: 320.0244$)$; UV (cyclohexane) $\mathrm{c}=$ $3.29 \times 10^{-4}, \lambda \max =206 \mathrm{~nm}(\mathrm{~A}=1.910, \varepsilon=5800), \lambda=230 \mathrm{~nm}(\mathrm{~A}=1.360, \varepsilon=4100)$, $257 \mathrm{~nm}(\mathrm{~A}=1.550, \varepsilon=4700), 280 \mathrm{~nm}(\mathrm{~A}=1.650, \varepsilon=5000) ; \mathrm{FT}-\mathrm{IR}\left(\mathrm{cm}^{-1}, \mathrm{CCl}_{4}\right) 3080$ (w, C-H), 1795 (s, - $\left.-\mathrm{CF}=\mathrm{CF}_{2}\right), 1630(\mathrm{~m}), 1446$ (s), 1321 (s), 1291 (s), 1238 (s), 1214 (s), 1195 (s) 1182 (s), 1172 (s), 1132 (s), 871 (m), 843 (m), 710 (m).

1b: Yield 83\%; GLPC purity 97\%; ${ }^{19} \mathrm{~F}$ NMR ( $\left.\mathrm{CDCl}_{3}\right)-59.7 \mathrm{ppm}\left(\mathrm{q}, 3 \mathrm{~F},-\mathrm{C} \mathrm{F}_{3}, \mathrm{Fa}\right),-60.1$ $\operatorname{ppm}\left(\mathrm{q}, 3 \mathrm{~F},-\mathrm{C}_{3}, \mathrm{Fb}\right),-63.7 \mathrm{ppm}\left(\mathrm{s}, 3 \mathrm{~F}, \phi-\mathrm{CF}_{3}, \mathrm{Fc}\right),-97.0 \mathrm{ppm}\left(\mathrm{dd}, 1 \mathrm{~F}\right.$, cis- $\left.\mathrm{CF}=\mathrm{C} \underline{F}_{2}, \mathrm{Fd}\right)$, $-111.0 \mathrm{ppm}\left(\mathrm{dd}, 1 \mathrm{~F}\right.$, trans $\left.-\mathrm{CF}=\mathrm{CF}_{2}, \mathrm{Fe}\right),-165.9 \mathrm{ppm}\left(\mathrm{dd}, 1 \mathrm{~F},-\mathrm{CF}=\mathrm{CF}_{2}, \mathrm{Ff}\right), \mathrm{U}_{\mathrm{ab}}=13 \mathrm{~Hz}$, $\left.J_{\mathrm{de}}=59 \mathrm{~Hz}, J_{\mathrm{df}}=32 \mathrm{~Hz}, J_{\mathrm{ef}}=118 \mathrm{~Hz}\right) ;{ }^{1} \mathrm{H} \mathrm{NMR}\left(\mathrm{CDCl}_{3}\right) 7.7 \mathrm{ppm}\left(\mathrm{d}, 2 \mathrm{H},{ }^{3} J_{\mathrm{HH}}=8 \mathrm{~Hz}\right) 7.4$ ppm (d, $\left.2 \mathrm{H},{ }^{3} \mathrm{JHH}_{\mathrm{HH}}=8 \mathrm{~Hz}\right) ;{ }^{13} \mathrm{C}\left\{{ }^{1} \mathrm{H}\right\}$ NMR $\left(\mathrm{CDCl}_{3}\right) 152.7 \mathrm{ppm}\left(\mathrm{ddd},-\mathrm{CF}=\underline{\mathrm{CF}}_{2},{ }^{1} J_{\mathrm{CF}}=294\right.$ $\left.\mathrm{Hz}, 280 \mathrm{~Hz},{ }^{2} J_{\mathrm{CF}}=45 \mathrm{~Hz}\right), 146.1 \mathrm{ppm}\left(\mathrm{m}, \phi-\underline{\mathrm{C}}\left(\mathrm{CF}_{3}\right)=\mathrm{C}\right), 138.1 \mathrm{ppm}(\mathrm{s}), 134.8 \mathrm{ppm}(\mathrm{s})$, $132.7 \mathrm{ppm}\left(\mathrm{q},-\underline{\mathrm{C}}\left(\mathrm{CF}_{3}\right),{ }^{2} J_{\mathrm{CF}}=33 \mathrm{~Hz}\right), 127.4 \mathrm{ppm}\left(\mathrm{q},-\mathrm{CF}_{3},{ }_{\mathrm{CF}}=266 \mathrm{~Hz}\right), 125.9 \mathrm{ppm}$ (s), $123.5 \mathrm{ppm}\left(\mathrm{q},-\underline{\mathrm{CF}}_{3},{ }^{1} \mathrm{~J}_{\mathrm{CF}}=272 \mathrm{~Hz}\right), 121.5 \mathrm{ppm}\left(\mathrm{m},-\underline{\mathrm{CF}}=\mathrm{CF}_{2}\right), 120.7 \mathrm{ppm}\left(\mathrm{q},-\underline{\mathrm{C}} \mathrm{F}_{3}\right.$, $1_{\mathrm{CF}}=266 \mathrm{~Hz}$ ); GC-MS $388\left(\mathrm{M}^{+}, 5\right), 369$ (M+-F, 16), 319 (70), 299 (34), 269 (77), 250 (100), 219 (59), 200 (71) HRMS $\left(\mathrm{C}_{13} \mathrm{H}_{4} \mathrm{~F}_{12}\right.$, calculated: 388.0121, observed:

388.0121); UV (cyclohexane) $\mathrm{c}=1.93 \times 10^{-4}, \lambda \max =212 \mathrm{~nm}(\mathrm{~A}=1.244, \varepsilon=6400), \lambda=$ $244 \mathrm{~nm}(\mathrm{~A}=0.607, \varepsilon=3100), 268 \mathrm{~nm}(\mathrm{~A}=0.560, \varepsilon=2900) ; \mathrm{FT}-\mathrm{IR}\left(\mathrm{cm}^{-1}, \mathrm{CCl}_{4}\right) 1795$ (s, $-\mathrm{CF}=\mathrm{CF}_{2}$ ), $1730(\mathrm{~m}, \mathrm{C}=\mathrm{C}), 1500(\mathrm{w}), 1440(\mathrm{w}), 1324$ (s) 1292 (s), 1241 (s), 1214 (s), 1194 (s), $1178(\mathrm{~s}), 1144(\mathrm{~s}), 1078(\mathrm{~m}), 723(\mathrm{~m})$.

1c: Yield 87\%; GLPC purity 96\%; ${ }^{19} \mathrm{~F}$ NMR ( $\left.\mathrm{CDCl}_{3}\right)-59.5 \mathrm{ppm}\left(\mathrm{q}, 3 \mathrm{~F},-\mathrm{CF}_{3}, \mathrm{Fa}\right),-60.4$ ppm (q, 3F, - $\left.\mathrm{CF}_{3}, \mathrm{Fb}\right),-99.5 \mathrm{ppm}\left(\mathrm{dd}, 1 \mathrm{~F}\right.$, cis- $\left.\mathrm{CF}=\mathrm{C} \underline{F}_{2}, \mathrm{Fc}\right),-112.3 \mathrm{ppm}(\mathrm{dd}, 1 \mathrm{~F}$, trans$\mathrm{CF}=\mathrm{CF} 2, \mathrm{Fd}),-164.9 \mathrm{ppm}\left(\mathrm{dd}, 1 \mathrm{~F},-\mathrm{CF}=\mathrm{CF}_{2}, \mathrm{Fe}\right),\left(\mathrm{Jab}_{\mathrm{ab}}=12 \mathrm{~Hz}, J_{\mathrm{cd}}=61 \mathrm{~Hz}, J_{\mathrm{ce}}=32 \mathrm{~Hz}, J_{\mathrm{de}}\right.$ $=117 \mathrm{~Hz}) ;{ }^{1} \mathrm{H} \mathrm{NMR}\left(\mathrm{CDCl}_{3}\right) 7.2 \mathrm{ppm}(\mathrm{d}, 2 \mathrm{H}), 6.9 \mathrm{ppm}(\mathrm{d}, 2 \mathrm{H}), 3.8 \mathrm{ppm}(\mathrm{s}, 3 \mathrm{H}) ;{ }^{13} \mathrm{C}$ $\left\{{ }^{1} \mathrm{H}\right\} \operatorname{NMR}\left(\mathrm{CDCl}_{3}\right) 161.8(\mathrm{~s}), 153.2 \mathrm{ppm}\left(\mathrm{ddd},-\mathrm{CF}=\mathrm{CF}_{2},{ }^{1} J_{\mathrm{CF}}=294 \mathrm{~Hz}, 280 \mathrm{~Hz},{ }^{2} J_{\mathrm{CF}}=46\right.$ $\mathrm{Hz}), 148.8 \mathrm{ppm}\left(\mathrm{q}, \phi-\underline{\mathrm{C}}\left(\mathrm{CF}_{3}\right)=\mathrm{C},{ }^{2} \mathrm{CFF}_{\mathrm{CF}}=34 \mathrm{~Hz}\right), 129.9 \mathrm{ppm}(\mathrm{s}), 125.2 \mathrm{ppm}(\mathrm{m},-$ $\left.\mathrm{C}\left(\mathrm{CF}_{3}\right)=\underline{\mathrm{C}}\left(\mathrm{CF}_{3}\right)-\mathrm{CF}\right), 124.0 \mathrm{ppm}(\mathrm{s}), 123.0 \mathrm{ppm}\left(\mathrm{ddd},-\underline{\mathrm{CF}}=\mathrm{CF}_{2},{ }^{1} J_{\mathrm{CF}}=236 \mathrm{~Hz},{ }^{2} \mathrm{JF}_{\mathrm{FF}}=55\right.$ $\mathrm{Hz}, 26 \mathrm{~Hz}), 121.2 \mathrm{ppm}\left(\mathrm{q},-\mathrm{CF}_{3},{ }^{1} J_{\mathrm{CF}}=274 \mathrm{~Hz}\right), 121.0 \mathrm{ppm}\left(\mathrm{q},-\underline{\mathrm{CF}}_{3},{ }^{1} J_{\mathrm{CF}}=275 \mathrm{~Hz}\right)$, $114.5 \mathrm{ppm}(\mathrm{s}), 55.3 \mathrm{ppm}(\mathrm{s})$; GC-MS $351\left(\mathrm{M}^{+}+1,17\right), 332\left(\mathrm{M}^{+}-\mathrm{F}, 9\right), 282(100), 231$ (30), 212 (19), 169 (24); HRMS $\left(\mathrm{C}_{13} \mathrm{H}_{7} \mathrm{~F}_{9} \mathrm{O}\right.$, calculated: 350.0353, observed: 350.0346); UV (cyclohexane) $\mathrm{c}=3.86 \times 10^{-4}, \lambda \max =226 \mathrm{~nm}(\mathrm{~A}=2.267, \varepsilon=5000), \lambda=$ $310 \mathrm{~nm}(\mathrm{~A}=1.143, \varepsilon=3000)$; FT-IR $\left(\mathrm{cm}^{-1}, \mathrm{CCl}_{4}\right) 3010(\mathrm{~m}), 2962(\mathrm{~m}), 2938(\mathrm{~m})$, $2912(\mathrm{~m}), 2842(\mathrm{~m}), 1787$ (s, -CF=CF $), 1609$ (s, C=C), 1513 (s), 1464 (m), 1443 (s), 1419 (s), 1351 (s), 1320 (s), 1290 (s), 1256 (s), 1239 (s), 1214 (s), 1194 (s), 1173 (s), 1132 (s), 874 (s), 828 (s).

1d: Yield 86\%; GLPC purity 98\%; ${ }^{19} \mathrm{~F}$ NMR ( $\left.\mathrm{CDCl}_{3}\right)-59.6 \mathrm{ppm}\left(\mathrm{q}, 3 \mathrm{~F},-\mathrm{C} \mathrm{F}_{3}, \mathrm{Fa}\right),-60.3$ ppm (q, 3F, $\left.-\underline{\mathrm{F}}_{3}, \mathrm{Fb}\right),-98.1 \mathrm{ppm}\left(\mathrm{dd}, 1 \mathrm{~F}\right.$, cis- $\left.\mathrm{CF}=\mathrm{C} \underline{\mathrm{F}}_{2}, \mathrm{Fc}\right),-111.5 \mathrm{ppm}(\mathrm{dd}, 1 \mathrm{~F}$, trans$\left.\mathrm{CF}=\mathrm{CF}_{2}, \mathrm{Fd}\right),-165.6 \mathrm{ppm}\left(\mathrm{dd}, 1 \mathrm{~F},-\mathrm{CF}=\mathrm{CF}_{2}, \mathrm{Fe}\right),\left(\mathrm{U}_{\mathrm{ab}}=13 \mathrm{~Hz}, J_{\mathrm{cd}}=61 \mathrm{~Hz}, J_{\mathrm{ce}}=32 \mathrm{~Hz}, J_{\mathrm{de}}\right.$ $=118 \mathrm{~Hz}) ;{ }^{1} \mathrm{H} \mathrm{NMR}\left(\mathrm{CDCl}_{3}\right) 7.4 \mathrm{ppm}\left(\mathrm{d}, 2 \mathrm{H},{ }^{3} J_{\mathrm{HH}}=8 \mathrm{~Hz}\right), 7.2 \mathrm{ppm}\left(\mathrm{d}, 2 \mathrm{H},{ }^{3} \mathrm{~J}_{\mathrm{HH}}=8 \mathrm{~Hz}\right)$; ${ }^{13} \mathrm{C}\left\{{ }^{1} \mathrm{H}\right\} \mathrm{NMR}\left(\mathrm{CDCl}_{3}\right) 153.1 \mathrm{ppm}\left(\mathrm{ddd},-\mathrm{CF}=\mathrm{CF}_{2},{ }^{1} J_{\mathrm{CF}}=295 \mathrm{~Hz}, 280 \mathrm{~Hz},{ }^{2} J_{\mathrm{CF}}=46 \mathrm{~Hz}\right)$, $147.2 \mathrm{ppm}\left(\mathrm{q}, \phi-\underline{\mathrm{C}}\left(\mathrm{CF}_{3}\right)=\mathrm{C},{ }^{2} J_{\mathrm{CF}}=34 \mathrm{~Hz}\right), 137.5 \mathrm{ppm}(\mathrm{s}), 130.2 \mathrm{ppm}(\mathrm{s}), 129.8 \mathrm{ppm}$ (s), $129.6 \mathrm{ppm}(\mathrm{s}), 126.3 \mathrm{ppm}\left(\mathrm{m},-\mathrm{C}\left(\mathrm{CF}_{3}\right)=\underline{\mathrm{C}}\left(\mathrm{CF}_{3}\right)-\mathrm{CF}\right), 122.2 \mathrm{ppm}$ (ddd, $-\underline{\mathrm{C} F}=\mathrm{CF}_{2},{ }^{1} \mathrm{JFF}_{\mathrm{CF}}$ $\left.=238 \mathrm{~Hz},{ }^{2} J_{\mathrm{CF}}=53 \mathrm{~Hz}, 26 \mathrm{~Hz}\right), 120.8 \mathrm{ppm}\left(\mathrm{q},-\underline{\mathrm{CF}}_{3},{ }^{1} \mathrm{~J}_{\mathrm{CF}}=275 \mathrm{~Hz}\right.$ ), $120.8 \mathrm{ppm}$ (two 
peak overlapping, q, $\left.-\underline{C F}_{3},{ }^{1} J_{\mathrm{CF}}=275 \mathrm{~Hz}\right)$; GC-MS $356\left(\mathrm{M}^{+}+2,6\right), 354\left(\mathrm{M}^{+}, 16\right), 319$ ( $\left.\mathrm{M}^{+}-\mathrm{Cl}, 97\right), 287$ (23), 285 (78), 269 (78), 250 (100), 235 (41), 200 (50); HRMS $\left(\mathrm{C}_{12} \mathrm{H}_{4} \mathrm{~F}_{9} \mathrm{Cl}\right.$, calculated: 353.9858 , observed: 353.9854$) ; \mathrm{UV}$ (cyclohexane) $\mathrm{c}=$ $4.24 \times 10^{-4}, \lambda \max =220 \mathrm{~nm}(\mathrm{~A}=1.526, \varepsilon=3600), \lambda=258 \mathrm{~nm}(\mathrm{~A}=0.587, \varepsilon=1380), \lambda$ $=284 \mathrm{~nm}(\mathrm{~A}=0.568, \varepsilon=1340) ;$ FT-IR $\left(\mathrm{cm}^{-1}, \mathrm{CCl}_{4}\right) 1780\left(\mathrm{~s},-\mathrm{CF}=\mathrm{CF}_{2}\right), 1620(\mathrm{w}), 1595$ (m), $1492(\mathrm{~m}), 1351$ (s), $1320(\mathrm{~s}), 1291$ (s), 1285 (s), 1266 (s), 1241 (s), 1233 (s), 1222 (s), 1212 (s), 1192 (s), 1182 (s), 1176 (s), 1163 (s), 1152 (s), 1134 (s), 1095 (s), $1019(\mathrm{~m}), 872(\mathrm{~m}), 820(\mathrm{~m})$.

1e: Yield 91\%; GLPC purity 96\%; ${ }^{19} \mathrm{~F}$ NMR ( $\left.\mathrm{CDCl}_{3}\right)-59.6 \mathrm{ppm}\left(\mathrm{q}, 3 \mathrm{~F},-\mathrm{CF}_{3}, \mathrm{Fa}\right),-60.4$ ppm (q, 3F, $\left.-\underline{\mathrm{F}}_{3}, \mathrm{Fb}\right),-99.4 \mathrm{ppm}\left(\mathrm{dd}, 1 \mathrm{~F}\right.$, cis- $\left.\mathrm{CF}=\mathrm{C} \underline{\mathrm{F}}_{2}, \mathrm{Fc}\right),-112.1 \mathrm{ppm}(\mathrm{dd}, 1 \mathrm{~F}$, trans$\left.\mathrm{CF}=\mathrm{CF}_{2}, \mathrm{Fd}\right),-165.1 \mathrm{ppm}\left(\mathrm{dd}, 1 \mathrm{~F},-\mathrm{CF}=\mathrm{CF}_{2}, \mathrm{Fe}\right),\left(\mathrm{U}_{\mathrm{ab}}=13 \mathrm{~Hz}, J_{\mathrm{cd}}=62 \mathrm{~Hz}, J_{\mathrm{ce}}=32 \mathrm{~Hz}, J_{\mathrm{de}}\right.$ $=117 \mathrm{~Hz}) ;{ }^{1} \mathrm{H}$ NMR $\left(\mathrm{CDCl}_{3}\right)$ 7.1-7.3 ppm $(\mathrm{m}, 4 \mathrm{H}), 2.4 \mathrm{ppm}(\mathrm{s}, 3 \mathrm{H}) ;{ }^{13} \mathrm{C}\left\{{ }^{1} \mathrm{H}\right\} \mathrm{NMR}$ $\left(\mathrm{CDCl}_{3}\right) 153.5 \mathrm{ppm}\left(\mathrm{ddd},-\mathrm{CF}=\underline{\mathrm{CF}}_{2},{ }^{1} J_{\mathrm{CF}}=294 \mathrm{~Hz}, 280 \mathrm{~Hz},{ }^{2} J_{\mathrm{CF}}=48 \mathrm{~Hz}\right), 148.7 \mathrm{ppm}(\mathrm{q}$, $\left.\phi-\underline{C}\left(\mathrm{CF}_{3}\right)=\mathrm{C},{ }^{2} J_{\mathrm{CF}}=34 \mathrm{~Hz}\right), 139.3 \mathrm{ppm}(\mathrm{s}), 132.0 \mathrm{ppm}(\mathrm{s}), 131.6 \mathrm{ppm}(\mathrm{s}), 129.0 \mathrm{ppm}$ (s), $128.7 \mathrm{ppm}(\mathrm{s}), 126.7 \mathrm{ppm}\left(\mathrm{m},-\mathrm{C}\left(\mathrm{CF}_{3}\right)=\underline{\mathrm{C}}\left(\mathrm{CF}_{3}\right)-\mathrm{CF}\right), 125.3 \mathrm{ppm}(\mathrm{s}), 122.5 \mathrm{ppm}$ (ddd, $\left.-\underline{C F}=\mathrm{CF}_{2},{ }^{1} J_{\mathrm{CF}}=240 \mathrm{~Hz},{ }^{2} J_{\mathrm{CF}}=55 \mathrm{~Hz}, 26 \mathrm{~Hz}\right), 121.2 \mathrm{ppm}\left(\mathrm{q},-\mathrm{CF}_{3},{ }^{1} J_{\mathrm{CF}}=276 \mathrm{~Hz}\right)$, $121.0 \mathrm{ppm}\left(\mathrm{q},-\mathrm{CF}_{3},{ }^{1} \mathrm{JCF}_{\mathrm{CF}}=276 \mathrm{~Hz}\right), 21.4 \mathrm{ppm}(\mathrm{s})$; GC-MS $334\left(\mathrm{M}^{+}, 21\right), 295$ (9), 265 (100), 245 (26), 215 (72), 196 (46), 146 (26); HRMS ( $\mathrm{C}_{13} \mathrm{H}_{7} \mathrm{~F}_{9}$, calculated: 334.0404, observed: 334.0396); UV (cyclohexane) $\mathrm{c}=2.54 \times 10^{-4}, \lambda \max =206 \mathrm{~nm}(\mathrm{~A}=0.587, \varepsilon=$ 2310), $\lambda=268 \mathrm{~nm}(\mathrm{~A}=0.026, \varepsilon=100) ; \mathrm{FT}-\mathrm{IR}\left(\mathrm{cm}^{-1}, \mathrm{CCl}_{4}\right) 3042(\mathrm{w}), 3031(\mathrm{w}), 2960$ (w, C-H), 2928 (w, C-H), 1789 (s, -CF=CF $), 1606$ (w), $1487(\mathrm{~m}), 1353$ (m), 1319 (s), 1294 (s), 1289 (s), 1251 (s), 1244 (s), 1230 (s), 1225 (s), 1219 (s), 1213 (s), 1210 (s), 1201 (s), 1190 (s), 1181 (s), 1172 (s), 1166 (s), 1162 (s), 1132 (s), 848 (m), 723 (m), $621(\mathrm{~m})$.

1f: Yield 52\%; mp 141-142 ${ }^{\circ} \mathrm{C}$; ${ }^{19} \mathrm{~F}$ NMR (376 MHz, JEOL GX-400, $\mathrm{CDCl}_{3}$ ) $-60.2 \mathrm{ppm}$ (qm, 3F, - $\mathrm{CF}_{3}, \mathrm{Fa}$ ), $-60.5 \mathrm{ppm}\left(\mathrm{qm}, 3 \mathrm{~F},-\mathrm{CF}_{3}, \mathrm{Fb}\right.$ ), $-98.6 \mathrm{ppm}\left(\mathrm{ddm}, 1 \mathrm{~F}\right.$, cis- $\mathrm{CF}=\mathrm{CF}_{2}, \mathrm{Fc}$ ), $-111.6 \mathrm{ppm}\left(\mathrm{dd}, 1 \mathrm{~F}\right.$, trans $\left.-\mathrm{CF}=\mathrm{CF}_{2}, \mathrm{Fd}\right),-166.6 \mathrm{ppm}\left(\mathrm{ddm}, 1 \mathrm{~F},-\mathrm{CF}=\mathrm{CF}_{2}, \mathrm{Fe}\right), \mathrm{Uab}_{\mathrm{ab}}=13$ $\left.\mathrm{Hz}, J_{\mathrm{cd}}=59 \mathrm{~Hz}, J_{\mathrm{ce}}=33 \mathrm{~Hz}, J_{\mathrm{de}}=117 \mathrm{~Hz}\right) ;{ }^{1} \mathrm{H} \mathrm{NMR}\left(400 \mathrm{MHz}\right.$, JEOL GX-400, $\left.\mathrm{CDCl}_{3}\right) 7.4$ ppm (s, 4H); ${ }^{13} \mathrm{C}\left\{{ }^{1} \mathrm{H}\right\}$ NMR (100 MHz, JEOL GX-400, $\left.\mathrm{CDCl}_{3}\right) 152.6 \mathrm{ppm}$ (ddd, $-\mathrm{CF}=\mathrm{CF}_{2}$, $\left.{ }^{1} J_{\mathrm{CF}}=297 \mathrm{~Hz}, 281 \mathrm{~Hz},{ }^{2} J_{\mathrm{CF}}=46 \mathrm{~Hz}\right), 146.2 \mathrm{ppm}\left(\mathrm{qm}, \phi-\underline{\mathrm{C}}\left(\mathrm{CF}_{3}\right)=\mathrm{C},{ }^{2} J_{\mathrm{CF}}=34 \mathrm{~Hz}\right), 133.5$ ppm (s), 128.3 ppm (s), 126.3 ppm (m, $\left.-\mathrm{C}\left(\mathrm{CF}_{3}\right)=\underline{\mathrm{C}}\left(\mathrm{CF}_{3}\right)-\mathrm{CF}\right), 121.7 \mathrm{ppm}$ (ddd, $\left.\underline{\mathrm{CF}}=\mathrm{CF}_{2},{ }^{1} J_{\mathrm{CF}}=236 \mathrm{~Hz},{ }^{2} J_{\mathrm{CF}}=54 \mathrm{~Hz}, 24 \mathrm{~Hz}\right), 120.3 \mathrm{ppm}\left(\mathrm{q},-\mathrm{CF}_{3},{ }^{1} J_{\mathrm{CF}}=269 \mathrm{~Hz}\right), 120.1$ $\operatorname{ppm}\left(\mathrm{q},-\mathrm{CF}_{3},{ }^{1} \mathrm{~J}_{\mathrm{CF}}=269 \mathrm{~Hz}\right) ; \mathrm{GC}-\mathrm{MS} 562\left(\mathrm{M}^{+}, 3\right), 493(26), 423(20), 355$ (40), 305 (42), 250 (42), $69\left(\mathrm{CF}_{3}, 100\right)$; HRMS $\left(\mathrm{C}_{18} \mathrm{H}_{4} \mathrm{~F}_{18}\right.$, calculated: 562.0026, observed: 562.0027); UV (cyclohexane) $\mathrm{c}=1.78 \times 10^{-4}, \lambda \max =216 \mathrm{~nm}(\mathrm{~A}=1.784, \varepsilon=10000), \lambda$ $=254 \mathrm{~nm}(\mathrm{~A}=1.554, \varepsilon=8700), \lambda=272 \mathrm{~nm}(\mathrm{~A}=1.526, \varepsilon=8600) ;$ FT-IR $\left(\mathrm{cm}^{-1}, \mathrm{CCl}_{4}\right)$ $1790\left(\mathrm{~m},-\mathrm{CF}=\mathrm{CF}_{2}\right), 1324$ (s), 1292 (s), 1267 (s), 1239 (s), 1217 (s), 1194 (s), 1177 (s), $1134(\mathrm{~s}), 867(\mathrm{w}), 721(\mathrm{w}), 715(\mathrm{w})$. 
trifluoro-3,4-bis(trifluoromethyl)-1,4-dihydronaphthalene (3a-e), anthracene (7) and phenanthrene (8) via photocyclization.

Compound $1(1.0 \mathrm{~g})$ was dissolved in hexane $(10 \mathrm{~mL})$ in a Rotaflo quartz tube.

After freezing this solution in liq. $\mathrm{N}_{2}$, the tube was degassed by a vacuum pump.

Then the solution was thawed and placed in the photoreactor $(254 \mathrm{~nm})$. After

reaction the solvent was evaporated and the obtained residue was purified via silica gel (70-230 mesh) chromatography (hexane) to yield $\mathbf{2}$ and $\mathbf{3}$. Yields are shown in

Table 2.

2a: Yield $430 \mathrm{mg}$ (46\%); $\mathrm{mp} 141-142{ }^{\circ} \mathrm{C}$; ${ }^{19} \mathrm{~F}$ NMR $\left(\mathrm{CDCl}_{3}\right)$-50.6 ppm (q, 3F, $\left.\mathrm{C}\left(\mathrm{CF}_{3}\right)=\mathrm{C}\left(\mathrm{CF}_{3}\right)-\mathrm{CF}=, \mathrm{Fa}\right),-53.3 \mathrm{ppm}\left(\mathrm{dq}, 3 \mathrm{~F},-\mathrm{C}\left(\mathrm{CF}_{3}\right)=\mathrm{C}\left(\mathrm{CF}_{3}\right)-\mathrm{CF}=, \mathrm{Fb}\right),-138.5 \mathrm{ppm}(\mathrm{d}$, $\left.1 \mathrm{~F},-\mathrm{C}\left(\mathrm{CF}_{3}\right)-\mathrm{CF}=\mathrm{CE}-, \mathrm{Fc}\right),-143.5 \mathrm{ppm}\left(\mathrm{dq}, 1 \mathrm{~F},-\mathrm{C}\left(\mathrm{CF}_{3}\right)-\mathrm{CE}=\mathrm{CF}-, \mathrm{Fd}\right), \mathrm{U}_{\mathrm{ab}}=17 \mathrm{~Hz}, \mathrm{Jd}_{\mathrm{bd}}=$ $\left.33 \mathrm{~Hz}, J_{\mathrm{cd}}=18 \mathrm{~Hz}\right) ;{ }^{1} \mathrm{H}$ NMR $\left(\mathrm{CDCl}_{3}\right) 8.3-7.7 \mathrm{ppm}(\mathrm{m}, 4 \mathrm{H}) ;{ }^{13} \mathrm{C}\left\{{ }^{1} \mathrm{H}\right\}$ NMR (acetone- $\mathrm{d}_{6}$ ) $148.9 \mathrm{ppm}\left(\mathrm{dd},-\underline{\mathrm{CF}}=\mathrm{CF}-{ }^{1} J_{\mathrm{CF}}=258 \mathrm{~Hz},{ }^{2} J_{\mathrm{CF}}=14 \mathrm{~Hz}\right), 144.4 \mathrm{ppm}\left(\mathrm{dd},-\mathrm{CF}=\underline{\mathrm{CF}}-{ }^{1} J_{\mathrm{CF}}=\right.$ $\left.255 \mathrm{~Hz},{ }^{2} \mathrm{JF}_{\mathrm{CF}}=14 \mathrm{~Hz}\right), 131.2 \mathrm{ppm}(\mathrm{s}), 130.3 \mathrm{ppm}(\mathrm{s}), 128.1 \mathrm{ppm}(\mathrm{bs}), 126.6 \mathrm{ppm}(\mathrm{m})$, $125.5 \mathrm{ppm}(\mathrm{m}), 124.0 \mathrm{ppm}\left(\mathrm{q},-\mathrm{CF}_{3},{ }^{1} \mathrm{~J}_{\mathrm{CF}}=276 \mathrm{~Hz}\right), 123.7 \mathrm{ppm}\left(\mathrm{qm},-\underline{\mathrm{C}}\left(\mathrm{CF}_{3}\right),{ }^{2} J_{\mathrm{CF}}=34\right.$ $\mathrm{Hz}), 123.1 \mathrm{ppm}\left(\mathrm{q},-\mathrm{CF}_{3},{ }^{1} \mathrm{JF}_{\mathrm{CF}}=277 \mathrm{~Hz}\right), 121.1 \mathrm{ppm}(\mathrm{bs}) ; \mathrm{GC}-\mathrm{MS} 301\left(\mathrm{M}^{+}+1,12\right), 300$ $\left(\mathrm{M}^{+}, 100\right), 423$ (28), 250 (100), 231 (40), 211 (12); HRMS $\left(\mathrm{C}_{12} \mathrm{H}_{4} \mathrm{~F}_{8}\right.$, calculated: 300.0185, observed: 300.0193$) ; \mathrm{UV}$ (cyclohexane) $\mathrm{c}=6.83 \times 10^{-5}, \lambda \max =228 \mathrm{~nm}(\mathrm{~A}$ $=2.966, \varepsilon=43400), \lambda=281 \mathrm{~nm}(\mathrm{~A}=0.4177, \varepsilon=6100), \lambda=334 \mathrm{~nm}(\mathrm{~A}=0.2945, \varepsilon=$ 4300); FT-IR ( $\left.\mathrm{cm}^{-1}, \mathrm{CCl}_{4}\right) 1650(\mathrm{~m}), 1616(\mathrm{~m}), 1467(\mathrm{~m}), 1402(\mathrm{~s}), 1379$ (s), 1315 (s), 1237 (s), 1211 (s), $1176(\mathrm{~s}), 1141(\mathrm{~s}), 952(\mathrm{w})$.

2b: Yield 53\%; GLPC purity 97\%; ${ }^{19} \mathrm{~F}$ NMR $\left(\mathrm{CDCl}_{3}\right)-51.4 \mathrm{ppm}\left(\mathrm{q}, 3 \mathrm{~F},-\mathrm{C}\left(\mathrm{CF}_{3}\right)=\mathrm{C}\left(\mathrm{CF}_{3}\right)-\right.$ $\mathrm{CF}=, \mathrm{Fa}),-54.4 \mathrm{ppm}\left(\mathrm{dq}, 3 \mathrm{~F},-\mathrm{C}\left(\mathrm{CF}_{3}\right)=\mathrm{C}\left(\mathrm{CF}_{3}\right)-\mathrm{CF}=, \mathrm{Fb}\right),-64.2 \mathrm{ppm}\left(\mathrm{s}, 3 \mathrm{~F},-\mathrm{CF}_{3}, \mathrm{Fc}\right)-$ $136.4 \mathrm{ppm}\left(\mathrm{d}, 1 \mathrm{~F},-\mathrm{C}\left(\mathrm{CF}_{3}\right)-\mathrm{CF}=\mathrm{CF}-\mathrm{Fd}\right),-139.6 \mathrm{ppm}\left(\mathrm{dq}, 1 \mathrm{~F},-\mathrm{C}\left(\mathrm{CF}_{3}\right)-\mathrm{CF}=\mathrm{CF}-, \mathrm{Fe}\right), \mathrm{Uab}_{\mathrm{ab}}$ $\left.=16 \mathrm{~Hz}, J_{\mathrm{be}}=32 \mathrm{~Hz}, J_{\mathrm{de}}=17 \mathrm{~Hz}\right) ;{ }^{1} \mathrm{H} \mathrm{NMR}\left(\mathrm{CDCl}_{3}\right) 8.5 \mathrm{ppm}(\mathrm{s}, 1 \mathrm{H}), 8.4 \mathrm{ppm}(\mathrm{d}, 1 \mathrm{H}$, $\left.{ }^{3} J_{\mathrm{HH}}=8 \mathrm{~Hz}\right), 7.9 \mathrm{ppm}\left(\mathrm{d}, 1 \mathrm{H},{ }^{3} \mathrm{~J}_{\mathrm{HH}}=8 \mathrm{~Hz}\right) ;{ }^{13} \mathrm{C}\left\{{ }^{1} \mathrm{H}\right\} \mathrm{NMR}\left(\mathrm{CDCl}_{3}\right) 148.9 \mathrm{ppm}(\mathrm{dd},-$ $\left.\underline{\mathrm{CF}}=\mathrm{CF}-,{ }^{1} J_{\mathrm{CF}}=263 \mathrm{~Hz},{ }^{2} J_{\mathrm{CF}}=14 \mathrm{~Hz}\right), 144.7 \mathrm{ppm}\left(\mathrm{dd},-\mathrm{CF}=\underline{\mathrm{CF}}-{ }^{1} J_{\mathrm{CF}}=262 \mathrm{~Hz},{ }^{2} J_{\mathrm{CF}}=14\right.$ $\mathrm{Hz}), 132.1 \mathrm{ppm}\left(\mathrm{q},-\mathrm{C}\left(\mathrm{CF}_{3}\right),{ }^{2} J_{\mathrm{CF}}=34 \mathrm{~Hz}\right), 128.8 \mathrm{ppm}(\mathrm{s}), 127.9 \mathrm{ppm}(\mathrm{m}), 126.6 \mathrm{ppm}$ (qm, $\left.-\underline{\mathrm{C}}\left(\mathrm{CF}_{3}\right)=\mathrm{C}\left(\mathrm{CF}_{3}\right)-\mathrm{CF}=,{ }^{2} J_{\mathrm{CF}}=39 \mathrm{~Hz}\right), 125.5 \mathrm{ppm}\left(\mathrm{d},-\mathrm{CF}=\mathrm{CF}-\underline{\mathrm{C}}-{ }^{2} J_{\mathrm{CF}}=14 \mathrm{~Hz}\right), 124.7$ ppm (s), $123.5 \mathrm{ppm}\left(\mathrm{q},-\mathrm{CF}_{3},{ }^{1} J_{\mathrm{CF}}=273 \mathrm{~Hz}\right), 122.8 \mathrm{ppm}\left(\mathrm{q},-\mathrm{CF}_{3},{ }^{1} J_{\mathrm{CF}}=274 \mathrm{~Hz}\right), 121.6$ $\left.\operatorname{ppm}\left(\mathrm{q},-\mathrm{CF}_{3},{ }^{1} \mathrm{~J}_{\mathrm{CF}}=277 \mathrm{~Hz}\right), 118.6 \mathrm{ppm}\left(\mathrm{q},-\mathrm{C}\left(\mathrm{CF}_{3}\right)=\mathrm{C}\left(\mathrm{CF}_{3}\right)-\underline{\mathrm{C}}-{ }^{3}\right]_{\mathrm{CF}}=5 \mathrm{~Hz}\right)$; GC-MS $368\left(\mathrm{M}^{+}, 92\right), 349$ (M+-F, 44), 318 (38), 299 (100), 249 (33), 229 (21), 211 (25); HRMS ( $\mathrm{C}_{13} \mathrm{H}_{3} \mathrm{~F}_{11}$, calculated: 368.0059, observed: 368.0059); UV (cyclohexane) $\mathrm{c}=$ $2.98 \times 10^{-5}, \lambda \max =224 \mathrm{~nm}(\mathrm{~A}=2.091, \varepsilon=70200), \lambda=276 \mathrm{~nm}(\mathrm{~A}=0.1920, \varepsilon=6400)$, $\lambda=336 \mathrm{~nm}(\mathrm{~A}=0.1374, \varepsilon=4600) ;$ FT-IR $\left(\mathrm{cm}^{-1}, \mathrm{CCl}_{4}\right) 3100(\mathrm{w}), 1680(\mathrm{~m}), 1648(\mathrm{~s})$, 
1631 (s), 1434 (s), 1393 (s), 1327 (s), 1291 (s), 1232 (s), 1208 (s), 1177 (s), 1145 (s), 1125 (s), 1074 (s), 1027 (s), 902 (s), 861 (s), 855 (m), 697 (m).

2c: Yield 50\%; GLPC purity 98\%; ${ }^{19} \mathrm{~F}$ NMR $\left(\mathrm{CDCl}_{3}\right)-51.3 \mathrm{ppm}\left(\mathrm{q}, 3 \mathrm{~F},-\mathrm{C}\left(\mathrm{CF}_{3}\right)=\mathrm{C}\left(\mathrm{CF}_{3}\right)-\right.$ $\mathrm{CF}=, \mathrm{Fa}),-53.7 \mathrm{ppm}\left(\mathrm{dq}, 3 \mathrm{~F},-\mathrm{C}\left(\mathrm{CF}_{3}\right)=\mathrm{C}\left(\mathrm{CF}_{3}\right)-\mathrm{CF}=, \mathrm{Fb}\right),-140.3 \mathrm{ppm}\left(\mathrm{d}, 1 \mathrm{~F},-\mathrm{C}\left(\mathrm{CF}_{3}\right)-\right.$ $\mathrm{CF}=\mathrm{CF}-, \mathrm{Fc}),-142.2 \mathrm{ppm}\left(\mathrm{dq}, 1 \mathrm{~F},-\mathrm{C}\left(\mathrm{CF}_{3}\right)-\mathrm{CF}=\mathrm{CF}-, \mathrm{Fd}\right), \mathrm{U}_{\mathrm{ab}}=16 \mathrm{~Hz}, J_{\mathrm{bd}}=32 \mathrm{~Hz}, J_{\mathrm{cd}}=$ $16 \mathrm{~Hz}) ;{ }^{1} \mathrm{H} \mathrm{NMR}\left(\mathrm{CDCl}_{3}\right) 8.0 \mathrm{ppm}(\mathrm{d}, 1 \mathrm{H}), 7.1 \mathrm{ppm}(\mathrm{m}, 2 \mathrm{H}), 3.9 \mathrm{ppm}(\mathrm{s}, 3 \mathrm{H}) ;{ }^{13} \mathrm{C}\left\{{ }^{1} \mathrm{H}\right\}$ $\operatorname{NMR}\left(\mathrm{CDCl}_{3}\right) 160.6 \mathrm{ppm}(\mathrm{s}), 147.1 \mathrm{ppm}\left(\mathrm{dd},-\underline{\mathrm{CF}}=\mathrm{CF}-,{ }^{1} J_{\mathrm{CF}}=257 \mathrm{~Hz},{ }^{2} J_{\mathrm{CF}}=14 \mathrm{~Hz}\right)$, $\left.144.3 \mathrm{ppm}\left(\mathrm{dd},-\mathrm{CF}=\underline{\mathrm{CF}}-{ }^{1}\right)_{\mathrm{CF}}=256 \mathrm{~Hz},{ }^{2} J_{\mathrm{CF}}=14 \mathrm{~Hz}\right), 128.1 \mathrm{ppm}(\mathrm{m}), 127.9 \mathrm{ppm}(\mathrm{s})$, $\left.124.0 \mathrm{ppm}\left(\mathrm{q},-\mathrm{CF}_{3},{ }^{1} \mathrm{~J}_{\mathrm{CF}}=276 \mathrm{~Hz}\right), 123.3 \mathrm{ppm}\left(\mathrm{q}, \underline{\mathrm{C}}-\mathrm{C}_{\left(\mathrm{CF}_{3}\right)}\right) \mathrm{C}\left(\mathrm{CF}_{3}\right)-,{ }^{2} J_{\mathrm{CF}}=36 \mathrm{~Hz}\right)$, $123.0 \mathrm{ppm}\left(\mathrm{q},-\underline{\mathrm{C}} \mathrm{F}_{3}, 1_{\mathrm{CF}}=276 \mathrm{~Hz}\right), 122.9 \mathrm{ppm}(\mathrm{s}), 122.3 \mathrm{ppm}(\mathrm{s}), 117.1 \mathrm{ppm}(\mathrm{m})$, $97.6 \mathrm{ppm}\left(\mathrm{q},{ }^{4}{ }_{\mathrm{CF}}=6 \mathrm{~Hz}\right), 55.8 \mathrm{ppm}(\mathrm{s})$; GC-MS $331\left(\mathrm{M}^{+}+1,11\right), 330\left(\mathrm{M}^{+}, 100\right), 311$ $\left(\mathrm{M}^{+}-\mathrm{F}, 21\right), 287$ (30), $268(10), 237$ (13), 218 (30); HRMS $\left(\mathrm{C}_{13} \mathrm{H}_{6} \mathrm{~F}_{8} \mathrm{O}\right.$, calculated: 330.0291, observed: 330.0280$)$; UV (cyclohexane) $\mathrm{c}=4.48 \times 10^{-5}, \lambda \max =245 \mathrm{~nm}(\mathrm{~A}$ $=2.426, \varepsilon=54000), \lambda=304 \mathrm{~nm}(\mathrm{~A}=0.3141, \varepsilon=7000) ; \mathrm{FT}-\mathrm{IR}\left(\mathrm{cm}^{-1}, \mathrm{CCl}_{4}\right) 2970(\mathrm{w})$, $2940(\mathrm{w}), 1720(\mathrm{w}), 1620(\mathrm{~m}), 1485(\mathrm{~m}), 1312$ (s), 1300 (s), 1279 (s), 1258 (s), 1245 (s), 1231 (s), 1222 (s), 1205 (s), 1171 (s), 1141 (m), 879 (m).

2d: Yield 59\%; GLPC purity >99\%; ${ }^{19} \mathrm{~F}$ NMR $\left(\mathrm{CDCl}_{3}\right)-51.4 \mathrm{ppm}(\mathrm{q}, 3 \mathrm{~F}$, $\left.\mathrm{C}\left(\mathrm{CF}_{3}\right)=\mathrm{C}\left(\mathrm{CF}_{3}\right)-\mathrm{CF}=, \mathrm{Fa}\right),-54.2 \mathrm{ppm}\left(\mathrm{dq}, 3 \mathrm{~F},-\mathrm{C}\left(\mathrm{CF}_{3}\right)=\mathrm{C}\left(\mathrm{CE}_{3}\right)-\mathrm{CF}=, \mathrm{Fb}\right),-138.2 \mathrm{ppm}(\mathrm{d}$, $\left.1 \mathrm{~F},-\mathrm{C}\left(\mathrm{CF}_{3}\right)-\mathrm{CF}=\mathrm{CE}-, \mathrm{Fc}\right),-140.1 \mathrm{ppm}\left(\mathrm{dq}, 1 \mathrm{~F},-\mathrm{C}\left(\mathrm{CF}_{3}\right)-\mathrm{CF}=\mathrm{CF}-, \mathrm{Fd}\right),\left(\mathrm{Jab}_{\mathrm{ab}}=16 \mathrm{~Hz}, \mathrm{Jbd}_{\mathrm{bd}}=\right.$ $\left.32 \mathrm{~Hz}, J_{\mathrm{cd}}=18 \mathrm{~Hz}\right) ;{ }^{1} \mathrm{H} \mathrm{NMR}\left(\mathrm{CDCl}_{3}\right) 8.2 \mathrm{ppm}\left(\mathrm{dm}, 1 \mathrm{H},{ }^{3} \mathrm{~J}_{\mathrm{HH}}=9 \mathrm{~Hz}\right), 8.0 \mathrm{ppm}(\mathrm{s}, 1 \mathrm{H})$, $7.5 \mathrm{ppm}\left(\mathrm{dm}, 1 \mathrm{H},{ }^{3} \mathrm{JHH}_{\mathrm{HH}}=9 \mathrm{~Hz}\right) ;{ }^{13} \mathrm{C}\left\{{ }^{1} \mathrm{H}\right\}$ NMR $\left(\mathrm{CDCl}_{3}\right) 147.3 \mathrm{ppm}\left(\mathrm{dd},-\underline{\mathrm{CF}}=\mathrm{CF}-,{ }^{1} \mathrm{JFF}_{\mathrm{F}}=\right.$ $\left.261 \mathrm{~Hz},{ }^{2} J_{\mathrm{CF}}=14 \mathrm{~Hz}\right), 144.6 \mathrm{ppm}\left(\mathrm{dd},-\mathrm{CF}=\underline{\mathrm{CF}}-,{ }^{1} J_{\mathrm{CF}}=259 \mathrm{~Hz},{ }^{2} J_{\mathrm{CF}}=14 \mathrm{~Hz}\right), 137.1 \mathrm{ppm}$ (s), $130.0 \mathrm{ppm}(\mathrm{s}), 128.0 \mathrm{ppm}(\mathrm{s}), 126.1 \mathrm{ppm}\left(\mathrm{d}, \mathrm{C}-\mathrm{CF}=\mathrm{CF}-,{ }^{2} J_{\mathrm{CF}}=12 \mathrm{~Hz}\right.$ ), $125.7 \mathrm{ppm}$ (s), $124.1 \mathrm{ppm}(\mathrm{m}), 122.3 \mathrm{ppm}\left(\mathrm{q},-\underline{\mathrm{CF}}_{3},{ }^{1} J_{\mathrm{CF}}=277 \mathrm{~Hz}\right), 121.9 \mathrm{ppm}\left(\mathrm{q},-\underline{C F}_{3},{ }^{1} J_{\mathrm{CF}}=277\right.$ $\mathrm{Hz}), 120.6 \mathrm{ppm}(\mathrm{m}), 119.4 \mathrm{ppm}(\mathrm{m})$; GC-MS $336\left(\mathrm{M}^{+}+2,43\right), 334\left(\mathrm{M}^{+}, 100\right), 315\left(\mathrm{M}^{+-}\right.$ F, 22), 299 (23), 284 (20), 265 (31); HRMS $\left(\mathrm{C}_{12} \mathrm{H}_{3} \mathrm{~F}_{8}{ }^{35} \mathrm{Cl}\right.$, calculated: 333.9796, observed: 333.9797); UV (cyclohexane) $\mathrm{c}=3.29 \times 10^{-5}, \lambda \max =236 \mathrm{~nm}(\mathrm{~A}=1.427, \varepsilon=$ 43400), $\lambda=290 \mathrm{~nm}(\mathrm{~A}=0.1561, \varepsilon=4700), \lambda=336 \mathrm{~nm}(\mathrm{~A}=0.0566, \varepsilon=1700) ; \mathrm{FT}-\mathrm{IR}$ $\left(\mathrm{cm}^{-1}, \mathrm{CCl}_{4}\right) 2990(\mathrm{w}), 2880(\mathrm{w}), 1790(\mathrm{w}), 1641(\mathrm{~m}), 1615(\mathrm{~m}), 1609(\mathrm{w}), 1495(\mathrm{~m})$, $1463(\mathrm{~m}), 1455(\mathrm{~m}), 1433(\mathrm{~m}), 1386(\mathrm{~m}), 1322(\mathrm{~m}), 1297(\mathrm{~s}), 1269(\mathrm{~s}), 1205$ (s), 1193 (s), 1178 (s), 1143 (s), 1027 (m), $858(\mathrm{~m}), 821(\mathrm{~m})$.

2e: GLPC Yield 25\%; ${ }^{19} \mathrm{~F}$ NMR $\left(\mathrm{CDCl}_{3}\right)-51.4 \mathrm{ppm}\left(\mathrm{m}, 3 \mathrm{~F},-\mathrm{C}\left(\mathrm{CF}_{3}\right)=\mathrm{C}\left(\mathrm{CF}_{3}\right)-\mathrm{CF}=, \mathrm{Fa}\right)$, $54.2 \mathrm{ppm}\left(\mathrm{m}, 3 \mathrm{~F},-\mathrm{C}\left(\mathrm{CF}_{3}\right)=\mathrm{C}\left(\mathrm{CF}_{3}\right)-\mathrm{CF}=, \mathrm{Fb}\right),-129.7 \mathrm{ppm}\left(\mathrm{m}, 1 \mathrm{~F},-\mathrm{C}\left(\mathrm{CF}_{3}\right)-\mathrm{CF}=\mathrm{CF}-, \mathrm{Fd}\right),-$ $143.0 \mathrm{ppm}\left(\mathrm{dq}, 1 \mathrm{~F},-\mathrm{C}\left(\mathrm{CF}_{3}\right)-\mathrm{CF}=\mathrm{CF}-, \mathrm{Fc}\right),\left(\mathrm{Jbc}_{\mathrm{bc}}=34 \mathrm{~Hz}, J_{\mathrm{cd}}=14 \mathrm{~Hz}\right) ;{ }^{1} \mathrm{H}$ NMR $\left(\mathrm{CDCl}_{3}\right)$ 7.9-7.4 ppm (m, 3H), $2.8 \mathrm{ppm}\left(\mathrm{d}, 3 \mathrm{H}, 5 \mathrm{JJF}_{\mathrm{HF}}=8 \mathrm{~Hz}\right)$; GC-MS $315\left(\mathrm{M}^{+}+1,13\right), 314\left(\mathrm{M}^{+}\right.$, 100), 295 (27), 263 (12), 245 (99), 224 (28), 175 (27).

2e': GLPC Yield 36\%;

${ }^{19} \mathrm{~F} \mathrm{NMR}\left(\mathrm{CDCl}_{3}\right)-51.4 \mathrm{ppm}\left(\mathrm{m}, 3 \mathrm{~F},-\mathrm{C}\left(\mathrm{CF}_{3}\right)=\mathrm{C}\left(\mathrm{CF}_{3}\right)-\mathrm{CF}=, \mathrm{Fa}\right),-54.0 \mathrm{ppm}(\mathrm{m}, 3 \mathrm{~F},-$ $\left.\mathrm{C}\left(\mathrm{CF}_{3}\right)=\mathrm{C}\left(\mathrm{CF}_{3}\right)-\mathrm{CF}=, \mathrm{Fb}\right),-139.2 \mathrm{ppm}\left(\mathrm{d}, 1 \mathrm{~F},-\mathrm{C}\left(\mathrm{CF}_{3}\right)-\mathrm{CF}=\mathrm{CF}-, \mathrm{Fc}\right),-144.1 \mathrm{ppm}(\mathrm{dq}, 1 \mathrm{~F}$, $\left.-\mathrm{C}\left(\mathrm{CF}_{3}\right)-\mathrm{CF}=\mathrm{CF}-, \mathrm{Fd}\right),\left(\mathrm{Jdd}_{\mathrm{bd}}=34 \mathrm{~Hz}, J_{\mathrm{cd}}=18 \mathrm{~Hz}\right) ;{ }^{1} \mathrm{H} \mathrm{NMR}\left(\mathrm{CDCl}_{3}\right)$ 7.9-7.4 ppm $(\mathrm{m}, 3 \mathrm{H})$, 
2.5 ppm (s, 3H); GC-MS $315\left(\mathrm{M}^{+}+1,13\right), 314\left(\mathrm{M}^{+}, 100\right), 295$ (M+-F, 27), 263 (13), 245 (98), 224 (32), 175 (18).

3a: Yield $190 \mathrm{mg}$ (19\%); GLPC purity 99\%; ${ }^{19} \mathrm{~F}$ NMR $\left(\mathrm{CDCl}_{3}\right)-60.7 \mathrm{ppm}(\mathrm{m}, 3 \mathrm{~F}$, $\left.\mathrm{CH}\left(\mathrm{CF}_{3}\right)-\mathrm{C}\left(\mathrm{CF}_{3}\right)=, \mathrm{Fa}\right),-69.6 \mathrm{ppm}\left(\mathrm{s}, 3 \mathrm{~F},-\mathrm{CH}\left(\mathrm{CF}_{3}\right)-\mathrm{C}\left(\mathrm{CF}_{3}\right)=\mathrm{CF}-, \mathrm{Fb}\right),-85.3 \mathrm{ppm}(\mathrm{dd}, 1 \mathrm{~F}$, $\left.=\mathrm{CF}-\underline{\mathrm{F}}_{2}-, \mathrm{Fc}\right),-101.3 \mathrm{ppm}\left(\mathrm{dd}, 1 \mathrm{~F},=\mathrm{CF}-\underline{\mathrm{F}}_{2^{-}}, \mathrm{Fd}\right),-114.9 \mathrm{ppm}\left(\mathrm{m}, 1 \mathrm{~F},=\mathrm{CF}-\mathrm{CF}_{2^{-}}, \mathrm{Fe}\right)$, $\left.U_{\mathrm{cd}}=304 \mathrm{~Hz}, J_{\mathrm{ce}}=30 \mathrm{~Hz}, J_{\mathrm{de}}=13 \mathrm{~Hz}\right) ;{ }^{1} \mathrm{H} \mathrm{NMR}\left(\mathrm{CDCl}_{3}\right)$ 7.9-7.5 ppm (m, 4H), $4.6 \mathrm{ppm}$ $\left(\mathrm{qm}, 1 \mathrm{H},-\mathrm{CH}\left(\mathrm{CF}_{3}\right)-,{ }^{3} J_{\mathrm{HF}}=7 \mathrm{~Hz}\right) ;{ }^{13} \mathrm{C}\left\{{ }^{1} \mathrm{H}\right\} \mathrm{NMR}\left(\mathrm{CDCl}_{3}\right) 156.7 \mathrm{ppm}\left(\mathrm{dt},=\underline{\mathrm{CF}}-\mathrm{CF}_{2^{-}},{ }^{1} J_{\mathrm{CF}}=\right.$ $\left.294 \mathrm{~Hz},{ }^{2} J_{\mathrm{CF}}=29 \mathrm{~Hz}\right), 131.6 \mathrm{ppm}(\mathrm{s}) 131.0 \mathrm{ppm}\left(\mathrm{t}, \underline{\mathrm{C}}-\mathrm{CF}_{2}-\mathrm{CF}=,{ }^{2} J_{\mathrm{CF}}=24 \mathrm{~Hz}\right), 130.3$ ppm (s), $129.6 \mathrm{ppm}(\mathrm{s}), 127.4 \mathrm{ppm}(\mathrm{s}), 126.2 \mathrm{ppm}(\mathrm{s}), 124.1 \mathrm{ppm}\left(\mathrm{q},-\mathrm{CF}_{3},{ }^{1} \mathrm{~J}_{\mathrm{CF}}=277\right.$ $\mathrm{Hz}), 121.8 \mathrm{ppm}\left(\mathrm{q},-\underline{\mathrm{CF}}_{3},{ }^{1} J_{\mathrm{CF}}=272 \mathrm{~Hz}\right), 111.1 \mathrm{ppm}\left(\mathrm{q},-\mathrm{CH}\left(\mathrm{CF}_{3}\right)-\mathrm{C}\left(\mathrm{CF}_{3}\right)=\mathrm{CF}-{ }^{2} J_{\mathrm{CF}}=30\right.$ $\mathrm{Hz}), 109.9 \mathrm{ppm}\left(\mathrm{ddd},=\mathrm{CF}-\mathrm{CF}_{2-},{ }^{1} \mathrm{JFF}_{\mathrm{F}}=239 \mathrm{~Hz}, 236 \mathrm{~Hz},{ }^{2} J_{\mathrm{CF}}=23 \mathrm{~Hz}\right), 43.7 \mathrm{ppm}(\mathrm{q},-$ $\left.\underline{\mathrm{CH}}\left(\mathrm{CF}_{3}\right)-,{ }^{2} J_{\mathrm{CF}}=31 \mathrm{~Hz}\right)$; GC-MS $320\left(\mathrm{M}^{+}, 13\right), 301$ (8), 251 (100), 232 (24), 201 (63), 182 (63); HRMS ( $\mathrm{C}_{12} \mathrm{H}_{5} \mathrm{~F}_{9}$, calculated: 320.0248, observed: 320.0249); UV (cyclohexane) $\mathrm{c}=3.28 \times 10^{-4}, \lambda \max =216 \mathrm{~nm}(\mathrm{~A}=1.565, \varepsilon=4800), \lambda=272 \mathrm{~nm}(\mathrm{~A}=$ 0.2328, $\varepsilon=710)$; FT-IR $\left(\mathrm{cm}^{-1}, \mathrm{CCl}_{4}\right) 2828(\mathrm{w}, \mathrm{C}-\mathrm{H}), 2853(\mathrm{w}), 1721(\mathrm{~m}), 1461(\mathrm{w})$, 1392 (m), 1315 (s), 1303 (s), 1260 (s), 1244 (s), 1184 (s), 1162 (s), 1138 (s), 1118 (s), $1059(\mathrm{~m}), 992(\mathrm{~m}), 658(\mathrm{~m})$.

3b: Yield 26\%; GLPC purity 95\%; ${ }^{19} \mathrm{~F} \mathrm{NMR}\left(\mathrm{CDCl}_{3}\right)-60.7 \mathrm{ppm}\left(\mathrm{m}, 3 \mathrm{~F},-\mathrm{CH}\left(\mathrm{CF}_{3}\right)-\right.$ $\left.\mathrm{C}\left(\mathrm{CF}_{3}\right)=, \mathrm{Fa}\right),-63.7 \mathrm{ppm}\left(\mathrm{s}, 3 \mathrm{~F},-\mathrm{CH}\left(\mathrm{CF}_{3}\right)-\mathrm{C}\left(\mathrm{CF}_{3}\right)=\mathrm{CF}-, \mathrm{Fb}\right),-69.2 \mathrm{ppm}\left(\mathrm{s}, 3 \mathrm{~F},-\mathrm{CF}_{3}, \mathrm{Fc}\right)$, $85.4 \mathrm{ppm}\left(\mathrm{dd}, 1 \mathrm{~F},=\mathrm{CF}-\underline{\mathrm{F}}_{2}-\mathrm{Fd}\right),-101.6 \mathrm{ppm}\left(\mathrm{dd}, 1 \mathrm{~F},=\mathrm{CF}-\underline{\mathrm{F}}_{2}-\mathrm{Fe}\right),-114.1 \mathrm{ppm}(\mathrm{m}, 1 \mathrm{~F}$, $\left.=\mathrm{CF}^{-}-\mathrm{CF}_{2}, \mathrm{Ff}\right),\left(\mathrm{J}_{\mathrm{aH}}=7 \mathrm{~Hz}, J_{\mathrm{de}}=306 \mathrm{~Hz}, J_{\mathrm{df}}=13 \mathrm{~Hz}, J_{\mathrm{ef}}=21 \mathrm{~Hz}\right) ;{ }^{1} \mathrm{H}$ NMR $\left(\mathrm{CDCl}_{3}\right) 8.1$ $\operatorname{ppm}(\mathrm{s}, 1 \mathrm{H}), 7.9 \mathrm{ppm}\left(\mathrm{d}, 1 \mathrm{H},{ }^{3} \mathrm{JHH}_{\mathrm{HH}}=7 \mathrm{~Hz}\right), 7.7 \mathrm{ppm}\left(\mathrm{d} 1 \mathrm{H},{ }^{3} \mathrm{~J}_{\mathrm{HH}}=7 \mathrm{~Hz}\right), 4.6 \mathrm{ppm}(\mathrm{q}, 1 \mathrm{H}$, $\left.-\mathrm{C} \underline{\mathrm{H}}\left(\mathrm{CF}_{3}\right)-,{ }^{3} J_{\mathrm{HF}}=7 \mathrm{~Hz}\right) ;{ }^{13} \mathrm{C}\left\{{ }^{1} \mathrm{H}\right\} \mathrm{NMR}\left(\mathrm{CDCl}_{3}\right) 156.4 \mathrm{ppm}\left(\mathrm{dt},=\underline{\mathrm{CF}}-\mathrm{CF}_{2-},{ }^{1} J_{\mathrm{CF}}=294 \mathrm{~Hz}\right.$, $\left.{ }^{2} J_{\mathrm{CF}}=27 \mathrm{~Hz}\right), 133.3 \mathrm{ppm}\left(\mathrm{q},-\underline{\mathrm{C}}\left(\mathrm{CF}_{3}\right),{ }^{2} J_{\mathrm{CF}}=34 \mathrm{~Hz}\right), 132.1 \mathrm{ppm}\left(\mathrm{t}, \underline{\mathrm{C}}-\mathrm{CF}_{2}-\mathrm{CF}=,{ }^{2} J_{\mathrm{CF}}=21\right.$ $\mathrm{Hz}), 131.1 \mathrm{ppm}(\mathrm{s}), 130.5 \mathrm{ppm}(\mathrm{s}), 128.4 \mathrm{ppm}(\mathrm{s}), 123.8 \mathrm{ppm}\left(\mathrm{q},-\underline{\mathrm{CF}}_{3},{ }^{1} J_{\mathrm{CF}}=272 \mathrm{~Hz}\right)$, $123.6 \mathrm{ppm}\left(\mathrm{q},-\underline{\mathrm{CF}}_{3},{ }^{1} \mathrm{JF}_{\mathrm{CF}}=277 \mathrm{~Hz}\right), 123.6 \mathrm{ppm}(\mathrm{s}), 121.3 \mathrm{ppm}\left(\mathrm{q},-\underline{\mathrm{CF}}_{3},{ }^{1} J_{\mathrm{CF}}=284 \mathrm{~Hz}\right)$, $111.1 \mathrm{ppm}\left(\mathrm{m},-\mathrm{CH}\left(\mathrm{CF}_{3}\right)-\underline{\mathrm{C}}\left(\mathrm{CF}_{3}\right)=\mathrm{CF}-\right), 109.3 \mathrm{ppm}\left(\mathrm{ddd},=\mathrm{CF}_{-} \mathrm{CF}_{2^{-}},{ }^{1} J_{\mathrm{CF}}=238 \mathrm{~Hz}, 235\right.$ $\left.\mathrm{Hz},{ }^{2} J_{\mathrm{CF}}=23 \mathrm{~Hz}\right), 43.8 \mathrm{ppm}\left(\mathrm{q},-\underline{\mathrm{CH}}\left(\mathrm{CF}_{3}\right)-{ }^{2} J_{\mathrm{CF}}=31 \mathrm{~Hz}\right) ; \mathrm{GC}-\mathrm{MS} 388\left(\mathrm{M}^{+}, 8\right), 369\left(\mathrm{M}^{+}-\mathrm{F}\right.$, 14), 319 (100), 300 (20), 269 (61), 265 (61), 200 (26); HRMS ( $\mathrm{C}_{13} \mathrm{H}_{4} \mathrm{~F}_{12}$, calculated: 388.0121, observed: 388.0118$)$; UV (cyclohexane) $\mathrm{c}=2.58 \mathrm{x} 10^{-4}, \lambda \max =226 \mathrm{~nm}(\mathrm{~A}$ $=0.893, \varepsilon=3500), \lambda=338 \mathrm{~nm}(\mathrm{~A}=0.075, \varepsilon=290) ; \mathrm{FT}-\mathrm{IR}\left(\mathrm{cm}^{-1}, \mathrm{CCl}_{4}\right) 1720(\mathrm{w})$, $1443(\mathrm{w}), 1388(\mathrm{w}), 1343(\mathrm{w}), 1316(\mathrm{~m}), 1282(\mathrm{~m}), 1246(\mathrm{~s}), 1232$ (s), $1198(\mathrm{~s})$, 1186 (s), 1165 (s), 1146 (s), 1124 (m), 1095 (m), 1067 (w), 911 (w).

3c: Yield 24\%; GLPC purity 95\%; ${ }^{19} \mathrm{~F}$ NMR $\left(\mathrm{CDCl}_{3}\right)-60.7 \mathrm{ppm}\left(\mathrm{m}, 3 \mathrm{~F},-\mathrm{CH}\left(\mathrm{CF}_{3}\right)-\right.$ $\left.\mathrm{C}\left(\mathrm{CF}_{3}\right)=, \mathrm{Fa}\right),-69.9 \mathrm{ppm}\left(\mathrm{s}, 3 \mathrm{~F},-\mathrm{CH}\left(\mathrm{CF}_{3}\right)-\mathrm{C}\left(\mathrm{CF}_{3}\right)=\mathrm{CF}-, \mathrm{Fb}\right),-85.4 \mathrm{ppm}\left(\mathrm{dd}, 1 \mathrm{~F},=\mathrm{CF}-\underline{F}_{2}-\right.$, Fc), $-101.4 \mathrm{ppm}\left(\mathrm{dd}, 1 \mathrm{~F},=\mathrm{CF}-\mathrm{F}_{2}-, \mathrm{Fd}\right),-115.5 \mathrm{ppm}\left(\mathrm{m}, 1 \mathrm{~F},=\mathrm{CF}_{-}-\mathrm{CF}_{2}-, \mathrm{Fe}\right), U_{\mathrm{cd}}=304 \mathrm{~Hz}$, $\left.J_{\mathrm{ce}}=23 \mathrm{~Hz}, J_{\mathrm{de}}=14 \mathrm{~Hz}\right) ;{ }^{1} \mathrm{H} \mathrm{NMR}\left(\mathrm{CDCl}_{3}\right) 7.4 \mathrm{ppm}\left(\mathrm{d}, 1 \mathrm{H},{ }^{3} J_{\mathrm{HH}}=9 \mathrm{~Hz}\right), 7.3 \mathrm{ppm}(\mathrm{s}), 7.1$ ppm $\left(\mathrm{d}, 1 \mathrm{H},{ }^{3} \mathrm{H}_{\mathrm{HH}}=9 \mathrm{~Hz}\right) 4.5 \mathrm{ppm}\left(\mathrm{qm}, 1 \mathrm{H},-\mathrm{C} \underline{\mathrm{H}}\left(\mathrm{CF}_{3}\right)-,{ }^{3} \mathrm{JFF}_{\mathrm{HF}}=7 \mathrm{~Hz}\right), 3.9 \mathrm{ppm}(\mathrm{s}, 3 \mathrm{H}) ;{ }^{13} \mathrm{C}$ $\left\{{ }^{1} \mathrm{H}\right\}$ NMR $\left(\mathrm{CDCl}_{3}\right) 161.2 \mathrm{ppm}(\mathrm{s}), 156.8 \mathrm{ppm}\left(\mathrm{dt},=\underline{\mathrm{CF}}-\mathrm{CF}_{2}-,{ }^{1} J_{\mathrm{CF}}=293 \mathrm{~Hz},{ }^{2} J_{\mathrm{CF}}=29=8\right.$ $\mathrm{Hz}), 132.2 \mathrm{ppm}\left(\mathrm{t}, \underline{\mathrm{C}}-\mathrm{CF}_{2}-\mathrm{CF}=,{ }^{2} J_{\mathrm{CF}}=24 \mathrm{~Hz}\right), 131.0 \mathrm{ppm}(\mathrm{s}), 124.0 \mathrm{ppm}\left(\mathrm{q},-\mathrm{CF}_{3},{ }^{1} J_{\mathrm{CF}}=\right.$ $282 \mathrm{~Hz}$ ), $121.3 \mathrm{ppm}\left(\mathrm{q},-\mathrm{CF}_{3},{ }^{1} J_{\mathrm{CF}}=275 \mathrm{~Hz}\right.$ ), $119.3 \mathrm{ppm}(\mathrm{bs}), 118.9 \mathrm{ppm}(\mathrm{s}), 109.7$ ppm (m), $110.2 \mathrm{ppm}(\mathrm{m}), 109.7 \mathrm{ppm}\left(\mathrm{ddd},=\mathrm{CF}_{-} \mathrm{CF}_{2-},{ }^{1} \mathrm{~J}_{\mathrm{CF}}=239 \mathrm{~Hz}, 236 \mathrm{~Hz},{ }^{2} \mathrm{JF}_{\mathrm{CF}}=24\right.$ $\mathrm{Hz}), 55.8 \mathrm{ppm}(\mathrm{s}), 43.3 \mathrm{ppm}\left(\mathrm{q},-\underline{\mathrm{CH}}\left(\mathrm{CF}_{3}\right)-{ }^{2}{ }^{2} \mathrm{JFF}_{\mathrm{C}}=31 \mathrm{~Hz}\right)$; GC-MS $350\left(\mathrm{M}^{+}, 22\right), 331$ 
(M+-F, 9), 281 (100), 231 (23), 212 (40), 169 (32); HRMS $\left(\mathrm{C}_{13} \mathrm{H}_{7} \mathrm{~F}_{9} \mathrm{O}\right.$, calculated: 350.0353, observed: 350.0358 ); UV (cyclohexane) $\mathrm{c}=3.14 \times 10^{-4}, \lambda \max =212 \mathrm{~nm}(\mathrm{~A}$ $=1.038, \varepsilon=3300), \lambda=242 \mathrm{~nm}(\mathrm{~A}=0.625, \varepsilon=2000), \lambda=334 \mathrm{~nm}(\mathrm{~A}=0.468, \varepsilon=$ 1500); FT-IR ( $\left.\mathrm{cm}^{-1}, \mathrm{CCl}_{4}\right) 2959(\mathrm{w}), 2930(\mathrm{w}), 1723(\mathrm{~m}), 1682(\mathrm{~m}), 1656(\mathrm{~m}), 1649$ (m), $1636(\mathrm{~m}), 1624(\mathrm{~m}), 1594(\mathrm{~m}), 1550(\mathrm{~m}), 1449(\mathrm{~m}), 1410(\mathrm{~s}), 1340(\mathrm{~m}), 1306$ (m), $1274(\mathrm{~m}), 1264(\mathrm{~m}), 1231(\mathrm{~m}), 1210(\mathrm{~s}), 1202$ (s), $1192(\mathrm{~s}), 1168(\mathrm{~s}), 1148$ (s), $1003(\mathrm{w}), 908(\mathrm{~m})$.

3d: Yield 14\%; GLPC purity 95\%; ${ }^{19} \mathrm{~F} \mathrm{NMR}\left(\mathrm{CDCl}_{3}\right)-60.8 \mathrm{ppm}\left(\mathrm{m}, 3 \mathrm{~F},-\mathrm{CH}\left(\mathrm{CF}_{3}\right)-\right.$ $\left.\mathrm{C}\left(\mathrm{CF}_{3}\right)=, \mathrm{Fa}\right),-69.5 \mathrm{ppm}\left(\mathrm{s}, 3 \mathrm{~F},-\mathrm{CH}\left(\mathrm{CF}_{3}\right)-\mathrm{C}\left(\mathrm{CF}_{3}\right)=\mathrm{CF}-, \mathrm{Fb}\right),-85.5 \mathrm{ppm}\left(\mathrm{dd}, 1 \mathrm{~F},=\mathrm{CF}-\mathrm{F}_{2}-\right.$, $\mathrm{Fc}),-101.5 \mathrm{ppm}\left(\mathrm{dd}, 1 \mathrm{~F},=\mathrm{CF}-\underline{-}_{2}-, \mathrm{Fd}\right),-114.7 \mathrm{ppm}\left(\mathrm{m}, 1 \mathrm{~F},=\mathrm{CF}^{-}-\mathrm{CF}_{2}-, \mathrm{Fe}\right), U_{\mathrm{FH}}=6 \mathrm{~Hz}$, $\left.J_{\mathrm{cd}}=305 \mathrm{~Hz}, J_{\mathrm{ce}}=23 \mathrm{~Hz}, J_{\mathrm{de}}=14 \mathrm{~Hz}\right) ;{ }^{1} \mathrm{H} \mathrm{NMR}\left(\mathrm{CDCl}_{3}\right) 7.8 \mathrm{ppm}(\mathrm{m}, 1 \mathrm{H}), 7.6 \mathrm{ppm}(\mathrm{dm}$, $\left.1 \mathrm{H},{ }^{3} J_{\mathrm{HH}}=8 \mathrm{~Hz}\right), 7.4 \mathrm{ppm}\left(\mathrm{d}, 1 \mathrm{H},{ }^{3} J_{\mathrm{HH}}=8 \mathrm{~Hz}\right), 4.5 \mathrm{ppm}\left(\mathrm{q}, 1 \mathrm{H},-\mathrm{CH}\left(\mathrm{CF}_{3}\right)-{ }^{3} J_{\mathrm{HF}}=6 \mathrm{~Hz}\right)$; ${ }^{13} \mathrm{C}\left\{{ }^{1} \mathrm{H}\right\}$ NMR $\left(\mathrm{CDCl}_{3}\right) 156.7 \mathrm{ppm}\left(\mathrm{dt},=\underline{\mathrm{CF}}-\mathrm{CF}_{2}-,{ }^{1} J_{\mathrm{CF}}=294 \mathrm{~Hz},{ }^{2} J_{\mathrm{CF}}=28 \mathrm{~Hz}\right), 137.3$ ppm (s), $133.4 \mathrm{ppm}\left(\mathrm{t}, \underline{\mathrm{C}}-\mathrm{CF}_{2}-\mathrm{CF}=,{ }^{2} J_{\mathrm{CF}}=28 \mathrm{~Hz}\right), 132.2 \mathrm{ppm}(\mathrm{s}), 131.2 \mathrm{ppm}(\mathrm{s}), 126.8$ ppm (m), $126.0 \mathrm{ppm}(\mathrm{s}), 124.0 \mathrm{ppm}\left(\mathrm{q},-\underline{\mathrm{CF}}_{3},{ }^{1} J_{\mathrm{CF}}=274 \mathrm{~Hz}\right), 121.3 \mathrm{ppm}\left(\mathrm{q},-\underline{C F}_{3},{ }^{1} J_{\mathrm{CF}}=\right.$ $274 \mathrm{~Hz}), 111.1 \mathrm{ppm}(\mathrm{m}), 109.4 \mathrm{ppm}\left(\mathrm{ddd},=\mathrm{CF}_{-} \mathrm{CF}_{2^{-}},{ }^{1} J_{\mathrm{CF}}=240 \mathrm{~Hz}, 239 \mathrm{~Hz},{ }^{2} J_{\mathrm{CF}}=24\right.$ $\mathrm{Hz}), 43.7 \mathrm{ppm}\left(\mathrm{q},-\underline{\mathrm{CH}}\left(\mathrm{CF}_{3}\right)-,{ }^{2} J_{\mathrm{CF}}=31 \mathrm{~Hz}\right) ; \mathrm{GC}-\mathrm{MS} 354\left(\mathrm{M}^{+}, 15\right), 335\left(\mathrm{M}^{+}-\mathrm{F}, 7\right), 287$ (41), 285 (100), 250 (22), 235 (44), 216 (54); HRMS $\left(\mathrm{C}_{12} \mathrm{H}_{4} \mathrm{~F}_{9}{ }^{35} \mathrm{Cl}\right.$, calculated: 353.9858, observed: 353.9844$)$ ) UV (cyclohexane) $\mathrm{c}=3.53 \times 10^{-4}, \lambda \max =220 \mathrm{~nm}$ (A $=2.013, \varepsilon=5700), \lambda=272 \mathrm{~nm}(\mathrm{~A}=0.172, \varepsilon=500), \lambda=282 \mathrm{~nm}(\mathrm{~A}=0.163, \varepsilon=460)$; FT-IR $\left(\mathrm{cm}^{-1}, \mathrm{CCl}_{4}\right) 1710(\mathrm{~m}), 1492(\mathrm{w}), 1430(\mathrm{w}), 1382(\mathrm{~m}), 1316(\mathrm{~m}), 1299(\mathrm{~m})$, 1278 (m), 1244 (s), 1232 (s), 1204 (s), 1196 (s), 1186 (s), 1165 (s), 1142 (s), 1122 (s), $1110(\mathrm{~s}), 1065(\mathrm{~s}), 855(\mathrm{~m})$.

3e: GLPC Yield 7\%; ${ }^{19} \mathrm{~F}$ NMR ( $\left.\mathrm{CDCl}_{3}\right)-60.9 \mathrm{ppm}(\mathrm{m}, 3 \mathrm{~F},-\mathrm{CF} 3, \mathrm{Fa}),-69.9 \mathrm{ppm}(\mathrm{s}, 3 \mathrm{~F}$, $\mathrm{CF}_{3}, \mathrm{Fb}$ ), $-93.8 \mathrm{ppm}\left(\mathrm{dd}, 1 \mathrm{~F},=\mathrm{CF}-\underline{\mathrm{F}}_{2}-, \mathrm{Fc}\right.$ ), $-95.8 \mathrm{ppm}\left(\mathrm{dd}, 1 \mathrm{~F},=\mathrm{CF}-\underline{\mathrm{F}}_{2}-, \mathrm{Fd}\right),-115.4 \mathrm{ppm}$ $\left(\mathrm{m}, 1 \mathrm{~F},=\mathrm{CF}-\mathrm{CF}_{2}-, \mathrm{Fe}\right),\left(U_{\mathrm{cd}}=309 \mathrm{~Hz}, J_{\mathrm{ce}}=22 \mathrm{~Hz}, J_{\mathrm{de}}=14 \mathrm{~Hz}\right) ;{ }^{1} \mathrm{H}$ NMR $\left(\mathrm{CDCl}_{3}\right)$ 7.7-7.3 ppm (m, 3H), 4.5 ppm (m, 1H) 2.6 ppm (m, 3H); GC-MS $334\left(\mathrm{M}^{+}, 58\right), 315\left(\mathrm{M}^{+}-\mathrm{F}, 18\right)$, 283 (60), 265 (100), 245 (26), 215 (27), 196 (81).

3e': GLPC Yield 18\%; ${ }^{19} \mathrm{~F}$ NMR ( $\left.\mathrm{CDCl}_{3}\right)-60.9 \mathrm{ppm}\left(\mathrm{m}, 3 \mathrm{~F},-\mathrm{CF}_{3}, \mathrm{Fa}\right),-69.6 \mathrm{ppm}(\mathrm{s}, 3 \mathrm{~F}$, $\left.-\mathrm{CF}_{3}, \mathrm{Fb}\right),-84.9 \mathrm{ppm}\left(\mathrm{dd}, 1 \mathrm{~F},=\mathrm{CF}-\underline{\mathrm{F}}_{2^{-}}, \mathrm{Fc}\right),-101.1 \mathrm{ppm}\left(\mathrm{dd}, 1 \mathrm{~F},=\mathrm{CF}-\underline{F}_{2^{-}}, \mathrm{Fd}\right),-115.1$ $\operatorname{ppm}\left(\mathrm{m}, 1 \mathrm{~F},=\mathrm{CF}-\mathrm{CF}_{2}-, \mathrm{Fe}\right),\left(\mathrm{Jae}_{\mathrm{ae}}=13 \mathrm{~Hz}, J_{\mathrm{cd}}=303 \mathrm{~Hz}, J_{\mathrm{ce}}=23 \mathrm{~Hz}\right) ;{ }^{1} \mathrm{H}$ NMR $\left(\mathrm{CDCl}_{3}\right) 7.7-$ $7.3 \mathrm{ppm}(\mathrm{m}, 3 \mathrm{H}), 4.5 \mathrm{ppm}(\mathrm{m}, 1 \mathrm{H}), 2.4 \mathrm{ppm}(\mathrm{m}, 3 \mathrm{H})$; GC-MS 334 (M+, 24), 265 (100), 246 (14), 215 (48), 196 (29), 146 (15).

7: Yield 7\%; mp 155-156 ${ }^{\circ} \mathrm{C} ;{ }^{19} \mathrm{~F}$ NMR (acetone- $\left.\mathrm{d}_{6}\right)-51.7 \mathrm{ppm}\left(\mathrm{q}, 6 \mathrm{~F},-\mathrm{C}\left(\mathrm{CF}_{3}\right)=\mathrm{C}\left(\mathrm{CF}_{3}\right)\right.$ $\mathrm{CF}=, \mathrm{Fa}),-54.6 \mathrm{ppm}\left(\mathrm{dq}, 6 \mathrm{~F},-\mathrm{C}\left(\mathrm{CF}_{3}\right)=\mathrm{C}\left(\mathrm{CF}_{3}\right)-\mathrm{CF}=, \mathrm{Fb}\right),-136.1 \mathrm{ppm}\left(\mathrm{d}, 2 \mathrm{~F},=\mathrm{C}\left(\mathrm{CF}_{3}\right)-\right.$ $\mathrm{CF}=\mathrm{CF}-, \mathrm{Fc}),-140.3 \mathrm{ppm}\left(\mathrm{qd}, 2 \mathrm{~F},=\mathrm{C}\left(\mathrm{CF}_{3}\right)-\mathrm{CF}=\mathrm{CF}-, \mathrm{Fd}\right), \mathrm{U}_{\mathrm{ab}}=16 \mathrm{~Hz}, J_{\mathrm{bd}}=32 \mathrm{~Hz}, J_{\mathrm{cd}}=$ $15 \mathrm{~Hz}$ ); ${ }^{1} \mathrm{H}$ NMR (acetone- $\left.\mathrm{d}_{6}\right) 9.3 \mathrm{ppm}(\mathrm{s}, 2 \mathrm{H}) ;{ }^{13} \mathrm{C}\left\{{ }^{1} \mathrm{H}\right\}$ NMR (acetone- $\left.\mathrm{d}_{6}\right) 149.0 \mathrm{ppm}$ $\left(\mathrm{dd},-\underline{C F}=\mathrm{CF}-,{ }^{1} J_{\mathrm{CF}}=262 \mathrm{~Hz},{ }^{2} J_{\mathrm{CF}}=14 \mathrm{~Hz}\right), 144.1 \mathrm{ppm}\left(\mathrm{dd},-\mathrm{CF}=\underline{\mathrm{CF}}-,{ }^{1} J_{\mathrm{CF}}=257 \mathrm{~Hz},{ }^{2} J_{\mathrm{CF}}=\right.$ $14 \mathrm{~Hz}), 126.2 \mathrm{ppm}(\mathrm{s}), 125.3 \mathrm{ppm}\left(\mathrm{d}, \underline{\mathrm{C}}-\mathrm{CF}=\mathrm{CF}-{ }^{2}{ }_{\mathrm{CF}}=13 \mathrm{~Hz}\right), 124.8 \mathrm{ppm}(\mathrm{m}), 123.7$ ppm (q, $\left.-\underline{C F}_{3},{ }^{1} J_{\mathrm{CF}}=271 \mathrm{~Hz}\right), 122.4 \mathrm{ppm}\left(\mathrm{q},-\underline{\mathrm{CF}}_{3},{ }^{1} J_{\mathrm{CF}}=276 \mathrm{~Hz}\right), 120.6 \mathrm{ppm}(\mathrm{s}) ; \mathrm{GC}-\mathrm{MS}$ $523\left(\mathrm{M}^{+}+1,17\right), 522\left(\mathrm{M}^{+}, 100\right), 503\left(\mathrm{M}^{+}-\mathrm{F}, 33\right), 472(25), 453$ (42), 315 (25); HRMS ( $\mathrm{C}_{18} \mathrm{H}_{2} \mathrm{~F}_{16}$, calculated: 521.9901, observed: 521.9924); UV (cyclohexane) $\mathrm{c}=5.42 \times 10$ 
$5, \lambda \max =260 \mathrm{~nm}(\mathrm{~A}=2.80, \varepsilon=51700), \lambda=205 \mathrm{~nm}(\mathrm{~A}=1.48, \varepsilon=27300), \lambda=400 \mathrm{~nm}$ $(\mathrm{A}=0.190, \varepsilon=3500), \lambda=430 \mathrm{~nm}(\mathrm{~A}=0.230, \varepsilon=4250) ; \mathrm{FT}-\mathrm{IR}\left(\mathrm{cm}^{-1}, \mathrm{CCl}_{4}\right) 1631(\mathrm{~m})$, $1549(\mathrm{w}), 1455(\mathrm{~m}), 1441(\mathrm{w}), 1430(\mathrm{w}), 1385(\mathrm{~m}), 1372(\mathrm{~m}), 1360(\mathrm{~m}), 1329(\mathrm{~s})$, 1294 (s), 1261 (s), 1232 (s), 1182 (s), 1146 (s), 1058 (w), 934 (w).

8: Yield 28\%; mp 129-130 ${ }^{\circ} \mathrm{C} ;{ }^{19} \mathrm{~F}$ NMR $\left(\mathrm{CDCl}_{3}\right)-50.0 \mathrm{ppm}\left(\mathrm{q}, 6 \mathrm{~F},-\mathrm{C}\left(\mathrm{CF}_{3}\right)=\mathrm{C}\left(\mathrm{CF}_{3}\right)-\right.$ $\mathrm{CF}=, \mathrm{Fa}),-54.5 \mathrm{ppm}\left(\mathrm{dq}, 6 \mathrm{~F},-\mathrm{C}\left(\mathrm{CF}_{3}\right)=\mathrm{C}\left(\mathrm{CF}_{3}\right)-\mathrm{CF}=, \mathrm{Fb}\right),-115.7 \mathrm{ppm}\left(\mathrm{bs}, 2 \mathrm{~F},-\mathrm{C}\left(\mathrm{CF}_{3}\right)-\right.$ $\mathrm{CF}=\mathrm{CF}-, \mathrm{Fc}),-134.2 \mathrm{ppm}\left(\mathrm{qm}, 2 \mathrm{~F},-\mathrm{C}\left(\mathrm{CF}_{3}\right)-\mathrm{CF}=\mathrm{CF}-, \mathrm{Fd}\right),\left(\mathrm{Uab}_{\mathrm{ab}}=16 \mathrm{~Hz}, \mathrm{Jd}_{\mathrm{bd}}=34 \mathrm{~Hz}\right) ;{ }^{1} \mathrm{H}$ NMR $\left(\mathrm{CDCl}_{3}\right) 8.2 \mathrm{ppm}(\mathrm{s}, 2 \mathrm{H}) ;{ }^{13} \mathrm{C}\left\{{ }^{1} \mathrm{H}\right\} \mathrm{NMR}\left(\mathrm{CDCl}_{3}\right) 150.1 \mathrm{ppm}\left(\mathrm{dd},-\underline{\mathrm{CF}}=\mathrm{CF}-,{ }^{1} \mathrm{JF}_{\mathrm{CF}}=\right.$ $\left.267 \mathrm{~Hz},{ }^{2} J_{\mathrm{CF}}=15 \mathrm{~Hz}\right), 147.6 \mathrm{ppm}\left(\mathrm{dm},-\mathrm{CF}=\underline{\mathrm{CF}}-{ }^{1} J_{\mathrm{CF}}=265 \mathrm{~Hz}\right), 128.2 \mathrm{ppm}(\mathrm{s}), 125.7$ ppm (m), $124.5 \mathrm{ppm}\left(\mathrm{dm}, \underline{\mathrm{C}}-\mathrm{CF}=\mathrm{CF}-,{ }^{2} J_{\mathrm{CF}}=31 \mathrm{~Hz}\right), 123.8 \mathrm{ppm}(\mathrm{m}), 122.7 \mathrm{ppm}(\mathrm{q},-$ $\left.\underline{\mathrm{CF}}_{3},{ }^{1} J_{\mathrm{CF}}=276 \mathrm{~Hz}\right), 122.6 \mathrm{ppm}\left(\mathrm{m},{ }^{2} J_{\mathrm{CF}}=33 \mathrm{~Hz}\right), 121.5 \mathrm{ppm}\left(\mathrm{q},-\underline{\mathrm{CF}}_{3},{ }^{1} J_{\mathrm{CF}}=275 \mathrm{~Hz}\right)$, $118.4 \mathrm{ppm}(\mathrm{bs})$; GC-MS $523\left(\mathrm{M}^{+}+1,18\right), 522\left(\mathrm{M}^{+}, 100\right), 503\left(\mathrm{M}^{+}-\mathrm{F}, 30\right), 472$ (27), 453 (33), 315 (13); HRMS $\left(\mathrm{C}_{18} \mathrm{H}_{2} \mathrm{~F}_{16}\right.$, calculated: 521.9901, observed: 521.9879); UV (cyclohexane) $c=5.43 \times 10^{-5}, \lambda \max =260 \mathrm{~nm}(\mathrm{~A}=2.17, \varepsilon=40000), \lambda=205 \mathrm{~nm}(\mathrm{~A}=$ $1.68, \varepsilon=310), \lambda=300 \mathrm{~nm}(\mathrm{~A}=0.415, \varepsilon=7650), \lambda=310 \mathrm{~nm}(\mathrm{~A}=0.415, \varepsilon=7650)$; FT-IR ( $\left.\mathrm{cm}^{-1}, \mathrm{CCl}_{4}\right) 1600(\mathrm{w}), 1493(\mathrm{w}), 1454(\mathrm{w}), 1434(\mathrm{~m}), 1416(\mathrm{~m}), 1352(\mathrm{~m})$, $1334(\mathrm{~m}), 1292(\mathrm{~m}), 1274(\mathrm{~m}), 1250(\mathrm{w}), 1221$ (s), 1200 (s), 1178 (s), 1145 (s), $1080(w), 1010(w), 902(w), 871(w)$.

\subsection{Conversion of $3 \boldsymbol{a}$ into $2 \boldsymbol{a}$ (NMR tube scale).}

The isolated 3a (60 mg, $0.20 \mathrm{mmol})$ was dissolved with $\mathrm{CDCl}_{3}(1.0 \mathrm{~mL})$ and DABCO (30 mg, $0.27 \mathrm{mmol}$ ) was added. After $30 \mathrm{~min}$ at room temperature, the reaction mixture was checked by ${ }^{19} \mathrm{~F}-\mathrm{NMR}$. Spectral data matched well with that obtained for the previously isolated $\mathbf{2 a}$. The conversion was quantitative. Additionally, the isolated 3a (100 mg) was dissolved in hexane $(1.0 \mathrm{~mL})$ and this solution was put into the photoreactor using the same method as in 3.6. After 2 days, no photoconversion to $\mathbf{2 a}$ was observed.

\subsection{Conversion of $3 \boldsymbol{e} / \mathbf{3} \boldsymbol{e}^{\prime}$ into $\mathbf{2} \boldsymbol{e} / \mathbf{2} \boldsymbol{e}^{\prime}$ (NMR tube scale).}

The mixture of $3 \mathbf{e} / 3 \mathbf{e}^{\prime}(80 \mathrm{mg}, 0.20 \mathrm{mmol})$ was dissolved with $\mathrm{CDCl}_{3}(1.0 \mathrm{~mL})$ and DBU (35 mg, $0.23 \mathrm{mmol}$ ) was added. After $30 \mathrm{~min}$ at room temperature, the 
reaction mixture was checked by ${ }^{19} \mathrm{~F}-\mathrm{NMR}$. This spectrum coincided with a previously isolated mixture of $\mathbf{2 e} / \mathbf{2} \mathbf{e}^{\prime}$.

\section{Conclusion}

In conclusion, readily prepared perfluoro cis-1,2-dimethyl butadienyl benzenes (1) underwent photocyclization to yield novel naphthalene and 1,4dihydronaphthalene derivatives. The isolated 1,4-dihydronaphthalene could be converted to their respective naphthalenes by base treatment but not photochemically. Competitive photochemical reactions indicate that varying the benzene substituent did not significantly affect reaction rate, suggesting that the mechanism does not involve electrophilic attack on the benzene as the ratedetermining step.

\section{Acknowledgement}

We wish to thank the National Science Foundation and the Air Force Office of Scientific Research for support of this work. M. Y. wishes to thank Nitto Denko Co., Ltd. (Japan) for the release time to pursue this research. D. J. wishes to thank Michael Sinnwell for help with manuscript preparation.

\section{References}

[1] D.M. Wiemers, D.J. Burton, J. Am. Chem. Soc., 108 (1986) 832-834; D.J Burton, Actualite Chimique, (1987) 142-146. [2] D.J. Burton, in: J.F. Liebman, A. Greenberg, W.R Dolbier Jr. (Eds.), Fluorine containing molecules: structure, reactivity, synthesis, and Applications; Wiley-VCH Verlag GmbH, New York, 1988 
[3] D.J. Burton, S.W Hansen, J. Am. Chem. Soc., 108 (1986) 4229-4230.

[4] T.D. Spawn, Ph.D. Thesis, University of Iowa, 1987

[5] D.J. Burton, C.R. Davis, in: Z. Rappoport, I. Marek (Eds), The Chemistry of organocopper compounds, Wiley, England, 2009, pp. 991-1032

[6] S.W. Hansen, Ph.D. Thesis, University of Iowa (1984).

[7] K.J. MacNeil, D.J. Burton. J. Org. Chem., 58 (1993) 4411-4417

[8] D.J. Burton, S.W. Hansen. J. Fluorine Chem., 130 (2009) 775-779

[9] R.D. Guneratne, D.J. Burton. J. Fluorine Chem., 98 (1999) 11-17

[10] E.P. Fokin, T.N. Gerasimova, T.V Fomenko, and N.V. Semikolenova, Zh. Org. Khim., 14 (1978) 834-841

[11] F.B. Mallory, C.W. Mallory, J. Org. Chem., 48 (1983) 526-532

[12] M. Gorsane, N. Defay, R.H. Martin, Bull. Soc. Chim. Belg., 94 (1985) 425-30

[13] D.C. Swenson, M. Yamamoto, D.J. Burton, Acta Cryst. C54 (1998) 846-849

[14] P.A. Morken, P.C. Bachand, D.C. Swenson, D.J. Burton, J. Am. Chem. Soc. 1993, $115,5430-5439$.

[15] S.W. Hansen, T.D. Spawn, D.J. Burton. J. Fluorine Chem., 35 (1987) 415-420 


\section{Graphical Abstract Synopsis}

The preparation of perfluoro cis-1,2-dimethyl butadienyl copper reagent and subsequent coupling with aryl iodides is described. Photocyclization of the corresponding butadienyl benzenes give novel naphthalene derivatives and 1,4dihydronaphthalenes. Additionally, naphthalene derivatives are prepared by treatment of 1,4-dihydronaphthalenes with DABCO. 


\section{Graphical Abstract}

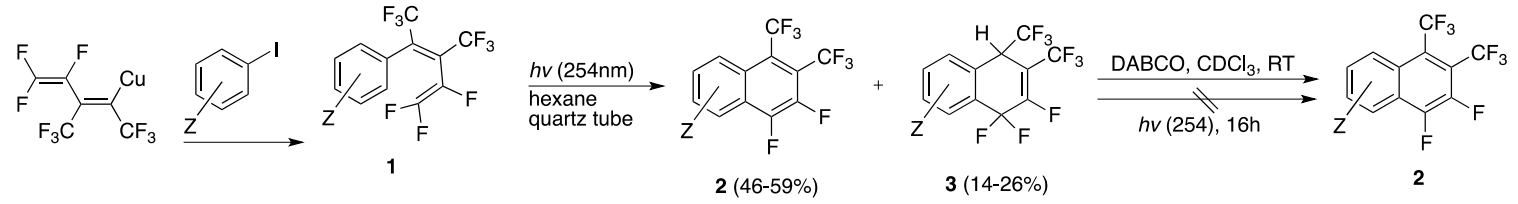

